\title{
Endogenous Recombinant Growth and Intellectual Property Rights*
}

\author{
Carla Marchese Simone Marsiglio $^{\ddagger} \quad$ Fabio Privileggi ${ }^{\S}$ \\ Giovanni B. Ramello
}

Forthcoming in Macroeconomic Dynamics

\begin{abstract}
We show that, even in a framework in which monopolistic exploitation of patents does not occur, patents still give rise to serious drawbacks. We rely on a recombinant growth framework that provides a stylized but clear description of the formation of knowledge externalities. In our setting a benevolent government buys immediately new patents in a competitive market and releases their contents for free. We show that inefficiencies nevertheless arise and welfare can be improved by correcting the market price through a taxsubsidy scheme. We characterize the (asymptotic) steady-state equilibrium, and some properties of the transitional path. We show that if certain conditions are met, then the economy will converge to its (asymptotic) balanced growth path, and along such a path growth will be independent of the policy parameter; conversely, transition dynamics are affected by the choice of the policy parameter. We then quantitatively analyze the effect of different policy interventions on welfare, and show that stricter tax (weaker appropriability) regimes lead to higher social welfare.
\end{abstract}

JEL Classification Numbers: K11; L16; L43; O31; O34; O41

Keywords: Endogenous Recombinant Growth; Intellectual Property Rights; Knowledge Production; Welfare

\section{Introduction}

Knowledge advances are a crucial ingredient in explaining economic growth. While the public good nature of knowledge is widely recognized, the literature on innovation alleges that appropriation opportunities - implemented via intellectual property rights (IPRs) — which entitle

\footnotetext{
${ }^{*}$ We are indebted to two anonymous referees for their constructive feedback which pushed us to rethink the model, clarifying its assumptions and results. We also wish to thank R. Boucekkine, A. Bucci, C. Garcia Peñalosa and the participants in the 12th Journées Louis-André Gérerd-Varet Conference in Public Economics (Aix-En-Provence, France, June 2013) for insightful and constructive discussion. All remaining errors and omissions are our own sole responsibility. Carla Marchese and Giovanni B. Ramello acknowledge the financial support from Università del Piemonte Orientale.

†Institute POLIS - DiGSPES, Università del Piemonte Orientale, Alessandria (Italy); carla.marchese@uniupo.it

${ }_{\ddagger}^{\ddagger}$ School of Accounting, Economics and Finance, University of Wollongong, Wollongong (NSW, Australia); simonem@uow.edu. au

$\S$ Dept. of Economics and Statistics "Cognetti de Martiis", Università di Torino, Lungo Dora Siena 100 A, 10153 Torino (Italy). Phone: +39-011-6702635; fax: +39-011-6703895; e-mail fabio.privileggi@unito.it

`Institute POLIS - DiGSPES, Università del Piemonte Orientale, Alessandria (Italy); and IEL \& BRICK, Collegio Carlo Alberto, Torino (Italy); giovanni.ramello@uniupo.it
} 
the right holder a legal monopoly over a fragment of knowledge, are indispensable institutions aimed at avoiding the market failure arising from the free-riding problem. Such a stylization, nevertheless, raises several concerns. Since a monopoly is unable to appropriate all social surplus, in general it does not supply the optimal incentive to inventors and authors, while different arrangements, such as rewards systems administered by the government, ${ }^{1}$ seem to a large extent better equipped to attain optimality (Arrow [1962], Shavell and van Ypersele [2001]). Further critical concerns relate to the incremental nature of knowledge production that recognize its role as an input; in such a case the above-cost pricing that monopoly entails will equally increase the cost of knowledge ${ }^{2}$ yielding ambiguous results both on social welfare and eventually on innovator profit (Scotchmer [1991], Bessen and Maskin [2009]). While most of the literature on endogenous growth in economies with IPRs has considered jointly the problems raised by the non-competitive nature of the market for knowledge on the one hand, and those deriving from the public good nature and the externalities stemming from knowledge production on the other, in this paper we aim at gaining further insights by focusing only on the second type of problems. We thus construct a stylized model in which the standard monopolistic exploitation problem is ruled out.

Our study builds on Weitzman's (1998) recombinant growth model, reformulated in a continuous time version by Tsur and Zemel (2007), which has the advantage with respect to other approaches of neatly describing the spillovers that characterize knowledge advances. In this framework it is assumed that the knowledge stock is endowed with some natural direct germinative property, which implies that when new research results are matched with old ones, then "hybrid seeds" capable of supporting further developments are produced. The combinatorial nature of knowledge accumulation in Weitzman's model represents a highly stylized but explicit description of the way in which externalities arise, in a vein that can be traced back either to natural Darwinian processes of evolution, or to social phenomena such as encounters, matchings and personal interactions that support knowledge growth. Weitzman's model, however, refers to a pure accumulation process in which knowledge production is centralized and governed by a social planner. We maintain the basic recombinant framework, but, differently from Weitzman (1998) in which the amount of resources allocated to knowledge production is exogenously given and from Tsur and Zemel (2007) in which this is entirely determined by a benevolent social planner, we explicitly introduce a decentralized system for knowledge production under the protection of patents. We assume that a benevolent government drives knowledge advances by immediately buying the patents on useful ideas newly produced by innovative firms which in turn exploit already available ideas. The government then releases new ideas for free, such that they can be exploited by the firms producing final consumption goods and also give rise to new seeds. Such a stylized institutional setting ${ }^{3}$ is substantially different from what generally considered in the traditional R\&D-based endogenous growth models. Its attractiveness with respect to more standard and simple alternatives (such as the abolition of the IPRs system and

\footnotetext{
${ }^{1}$ Public funding in various forms does play today an important and not substitutable role in stimulating innovations, as it stably counts for at least one third of gross domestic R\&D expenditures in many developed economies, US included (OECD [2016]).

${ }^{2}$ Besides the direct cost increase connected with the higher price of knowledge monopolized inputs (Scotchmer [1991]) there is an indirect one connected with transaction costs incurred for searching, contracting and buying the licenses for all the dispersed fragments of proprietary knowledge needed as inputs. When there are complementary inputs an adverse outcome, the so called 'tragedy of anticommons' can arise (Heller and Eisenberg [1998], Ziedonis [2004]).

${ }^{3}$ Note that such a setting seems to be consistent with some experiences documented by economic history. In fact, governments have actually sometimes bought patents, and proposals have been advanced for adopting systematically such an approach. On this issue see for example p. 528 in Shavell and van Ypersele (2001).
} 
the direct subsidization of research) rests on the activation of a transparent free-entry market for the production of new knowledge. The role of patents as a way of encouraging disclosure of inventions (as an alternative to trade secrecy) has often been stressed in the literature (Denicolò and Franzoni, 2004). In our framework disclosure would be immediate, while the role of the public sector would be confined to the collection of resources and to the organization of the IPRs system, while the potential drawbacks associated with bureaucratic interventions in productive activities would be avoided.

As in Weitzman we assume that knowledge behaves as a homogeneous good when transferred to the final goods production; while it must be new and distinguishable when patented. The buyer, i.e., the benevolent government, behaves as a price taker in the knowledge market. Hence, the drawbacks caused by standard forms of monopolistic exploitation of IPRs cannot arise, since there is competition between suppliers and no monopsonistic exploitation by the government. However, the appropriability of knowledge via IPRs still involves inefficiencies, because the equilibrium price in the market for knowledge turns out to be distorted due to the presence of externalities pertaining to the seeds formation. Moreover, innovative firms selling IPRs to the government at equilibrium market prices enjoy rents, as they privately appropriate the benefits stemming from the seeds process. Rents, however, attract potential challengers of the IPRs, and thus in order to prevent them new knowledge producers must waste resources in legal and administrative expenditures. However, the power of the germinative property of knowledge implies that asymptotically economies with this IPRs system converge to a socially efficient outcome in terms of growth. We show that, in order to increase welfare, the government can resort in the transitory period to a tax-subsidy scheme for correcting the equilibrium market price. A subsidy financed by taxation on the rents of the IPRs owners or the limitation of rent appropriability granted by IPRs can redress the aforementioned drawbacks. Our results thus show the relevance of corrective government interventions (via taxation/subsidy schemes or modifications of the IPRs owner entitlements), which turn out to be desirable even within a scenario that rules out the most commonly recognized potential problems raised by the IPRs system. Moreover, our results provide motivations for taxing rents from IPRs, a policy option that seems relevant in practice but which does not fit well into the standard endogenous growth approach.

The paper proceeds as follows. In Section 2 we introduce our model specification featuring a decentralized market for knowledge production based on a basic IPRs system and the possibility of government intervention to contain inefficiencies by applying a certain tax-subsidy scheme. In Section 3 we discuss short and long-run equilibria, showing that if certain conditions are met the economy converges towards an asymptotic balanced growth path along which the growth rate is not affected by the government policy, while the transition equilibrium depends on the policy parameter. Section 4 presents our model's specification which allows us to discuss and analyze in more depth the transitional dynamics. Section 5 contains our quantitative results: we basically show here that an active government intervention is beneficial, as welfare increases with the policy parameter. Section 6 comments on the policy implications of our model and presents concluding remarks. All mathematical proofs and a description of the numerical techniques employed are postponed in Appendices A and B, respectively.

\section{The Model}

Our model is a Ramsey-type model of growth with endogenous creation of knowledge according to a recombinant process. As we focus mainly on the effects of knowledge-related policies on growth patterns and welfare, for the sake of simplicity we assume that the only durable good is 
the stock of knowledge $A$; in other words, there is no (physical) capital accumulation and the only asset in the economy is represented by knowledge.

The economy is composed by households, firms and the government. Households receive a wage for the labor supplied to the production sector, pay a lump-sum tax and purchase a consumption good. Moreover, they socially interact and combine the stock of existing ideas with the newly produced ones, thus producing seed ideas for free. There are two types of firms: one producing the final consumption good and one performing knowledge creation activities. The government collects taxes from households and invests the proceeds into knowledge creation; moreover, it resorts to a tax-subsidy scheme applied to the latter type of firms in order to correct the inefficiencies generated by a basic IPRs system, as will be explained in the following.

\subsection{Recombinant Knowledge Creation}

As in the Tsur and Zemel (2007) continuous time version of Weitzman (1998) model, the new knowledge production function is defined as

$$
\dot{A}(t)=H(t) \eta\left[\frac{J(t)}{H(t)}\right]
$$

where $\dot{A}(t)$ denotes the time derivative of the stock of knowledge $A(t), J(t)$ the amount of resources employed in knowledge production, $H(t)$ the number of hybrid seed ideas, and $\eta(\cdot)$ is the probability of obtaining a successful idea from each hybridization (matching) satisfying the following assumption, as in Weitzman (1998).

A. 1 The success probability function $\eta: \mathbb{R}_{+} \rightarrow[0,1]$ is independent of time and is such that $\eta^{\prime}(x)>0, \eta^{\prime \prime}(x)<0, \eta(0)=0$ and $\lim _{x \rightarrow \infty} \eta(x) \leq 1$; moreover, it will be assumed that $\eta^{\prime}(0) \equiv \lim _{x \rightarrow 0^{+}} \eta^{\prime}(x)<+\infty$ and $\eta^{\prime \prime}(0) \equiv \lim _{x \rightarrow 0^{+}} \eta^{\prime \prime}(x)>-\infty$.

Note also that (1) can be interpreted as a standard production function from inputs to output, characterized by homogeneity of degree one and thus constant returns to scale. According to this widely used point of view - that we will adopt in the sequel - aggregate knowledge creation is presented as a deterministic process, even if, of course, there may be idiosyncratic uncertainty.

The necessary smoothness assumption required in a continuous-time setting implies that $\dot{A}(t)$ has the same value both while looking forward to the new output - equation (1) - and while looking backward, i.e., to the formation of seed ideas, given by

$$
H(t)=C_{m}^{\prime}[A(t)] \dot{A}(t),
$$

where $C_{m}^{\prime}(A)$ denotes the derivative of the number of different combinations of $m$ elements as a function of the stock ${ }^{4} A$ (Tsur and Zemel [2007], Privileggi [2010; 2011]); that is, within this approach both the seed production in (2) and the production of new ideas in (1) are referred (as a limit) to the same time instant. Hence, when (2) is substituted into (1), it turns out that the law of motion of knowledge at every instant is

$$
\dot{A}(t)=\frac{J(t)}{\varphi[A(t)]},
$$

\footnotetext{
${ }^{4}$ Specifically: $C_{m}(A)=A ! /[m !(A-m) !]$. For example, $C_{2}(A)=A(A-1) / 2$.
} 
where

$$
\varphi(A)=C_{m}^{\prime}(A) \eta^{-1}\left[\frac{1}{C_{m}^{\prime}(A)}\right]
$$

is the expected unit cost of knowledge production as a function of the stock of knowledge $A$, converging to the constant $1 / \eta^{\prime}(0)$ as $A \rightarrow \infty$; note that $1 / \eta^{\prime}(0)$ is strictly positive under Assumption A.1.

In the following we focus on a decentralized framework, where a benevolent regulator decides how many resources to convey to buying new knowledge on the market. The seed ideas available at time $t$, defined by (2), arise out of an externality associated with the process of knowledge creation, and do not command any specific compensation. The R\&D industry thus exploits $H$ for free and combines it with $J$ to produce new knowledge $\dot{A}$.

\subsection{Households}

To further simplify the analysis, we abstract from population growth and the population size is normalized to unity. We adopt the standard assumption that all households have an increasing and concave utility function, $u^{\prime}(c)>0, u^{\prime \prime}(c)<0$ where $c$ is consumption. A social planner chooses consumption in order to maximize social welfare, that is the infinite discounted sum of utilities, with a rate of time preference denoted by $\rho>0$. As we shall show, all the firms operate in competitive markets and thus their profits are zero. Moreover, as the new knowledge is being purchased by the government and released for free in the economy, there is no possession of the only asset (knowledge) by households and, in turn, no asset accumulation. Hence, they face only an instantaneous constraint: $c(t) \leq w(t)-G(t)$, where $w(t)$ is the wage households receive for supplying labor and $G(t)$ denotes the lump-sum tax they pay to the government.

\section{$2.3 \quad F$-firms}

In the final consumption good sector firms ( $F$-firms) are competitive and operate in a standard neoclassical framework: at each instant $F$-firm $i$ employs a composite intermediate good, $X_{i}$, and knowledge-augmented labor to produce a composite consumption good according to a constant returns to scale production function, $Y_{i}=F\left(X_{i}, A L_{i}\right)$, taking as given the labor wage, $w$, and the stock of knowledge, $A$, which is supplied by the government for free. Because they all use the same technology and face the same market conditions, they also employ the same intermediate good/labor ratio; hence the subscript $i$ can be dropped and per capita output turns out to be $y=Y / L=F(x, A) / L$, where $x=X / L$. Moreover, as $F(\cdot, \cdot)$ shows constant return to scale, all identical $F$-firms pay the production factors (the intermediate good $x$ and labor) their marginal cost (the numeraire and the wage $w$ respectively) and earn zero profit. Recalling the assumption that $L(t) \equiv 1$ for all $t$, it is convenient to rewrite the aggregate output as

$$
Y=A f\left(\frac{x}{A}\right)
$$

where $f(\cdot)=F(\cdot, 1)$ is increasing and concave.

It is assumed that new knowledge can be directly transferred to production of final goods (through, e.g., organizational and process innovations), while such transfer implies a rescaling to take into account the effectiveness in terms of output augmentation (Weitzman [1998], p. 344), as will be clarified later on. The possibility of supplying directly knowledge contents to $F$-firms seems realistic because nowadays patents refer more and more to procedures, routines, marketing models, etc., i.e., to dematerialized contents that $F$-firms can directly implement in their activity (Chantrel et al. [2012], Marchese and Privileggi (2017)). 


\section{$2.4 \quad R \& D$-firms and the Innovations' Market Equilibrium}

There is a large number $n$ of firms creating new knowledge ( $R \& D$-firms) which exploit the recombinant technology described in (1), and there is free entry in the market. The government buys immediately newly patented knowledge and releases it for free. While on the demand side the government is a monopsonist, it is assumed that it is benevolent and behaves as a pricetaker. Seed ideas $H$ arise for free as a result of the externality generated by the knowledge creation process, in which the new knowledge $\dot{A}$ released by the government is combined with the already existing knowledge stock $A(t)$ according to $(2) .^{5}$ While new knowledge is a public good (it is non-rival when used in the production of either final goods or new seeds), seeds are rival, that is, no new knowledge could be produced by processing the same seeds again. The free availability of seeds in a market with free entry might entail a tragedy of the commons. The IPRs system helps in avoiding this, since it assigns the property right on each specific bit of new knowledge to the patent holder, and each patented bit of knowledge can be traced back to a specific subset of seeds (while instead in the case of commons a homogeneous good is produced and the latter cannot be traced back to a specific part of the pool). However, as the IPRs system only indirectly supports the de-facto private appropriation of the seeds that led to an invention, the owner of a patent is exposed to frequent controversies. $R \& D$-firms must thus bear the fixed (instantaneous) cost $\mu_{i}$ to deter potential challengers. ${ }^{6}$

Each $R \& D$-firm $i$ takes the price of new knowledge $\psi$ as given, and, in order to obtain a patentable result $\dot{A}_{i}$, aims at exploiting a share $\bar{H}_{i}=H / n$ of the available seeds. It takes $\bar{H}_{i}$ as exogenously determined and given. That is, each $R \& D$-firm does not anticipate that its own research activity and that of the other firms will expand $H$ according to equation (2). Since seeds are available for free, the firm's $i$ instantaneous profit is given by

$$
\pi_{i}=\psi \dot{A}_{i}-J_{i}-\mu_{i}
$$

where $\mu_{i}$ is a fixed cost it bears to prevent legal challenges. In view of (1), each $R \& D$-firm profit can be rewritten as

$$
\pi_{i}\left(J_{i}\right)=\psi \bar{H}_{i} \eta\left(J_{i} / \bar{H}_{i}\right)-J_{i}-\mu_{i} .
$$

Strict concavity of $\eta(\cdot)$ and the fact that $\eta(\cdot) \leq 1$ [that is, $\psi \bar{H}_{i} \eta\left(J_{i} / \bar{H}_{i}\right)$ is bounded from above by $\psi \bar{H}_{i}$, which is independent of $J_{i}$ ] dictated by the smoothness assumption A.1 are enough for (6) to have a unique (interior) maximum $J_{i}^{*}$ provided that $\pi_{i}^{\prime}(0)=\psi \eta^{\prime}(0)-1>0$, that is, whenever

$$
\psi>1 / \eta^{\prime}(0) .
$$

We shall see that condition (7) is always satisfied under Assumption A.1 (see Proposition 2 below). Then, FOC on (6) with respect to $J_{i}$ yields the optimal amount of physical resources that the firm chooses to employ in knowledge production for given $\bar{H}_{i}$ seed ideas and price $\psi$ :

$$
J_{i}^{*}=\bar{H}_{i}\left(\eta^{\prime}\right)^{-1}(1 / \psi),
$$

\footnotetext{
${ }^{5}$ The formation of seed ideas may also be interpreted as arising from costless social interactions. The innovation (Ramello [2005; 2011] and economic history (Rosenberg [1976], Ceruzzi [2003]) literature stress that knowledge may be the results of also non-market mechanisms. This might explain why the stock of knowledge (specifically, seed ideas) may rise autonomously, providing thus some foundation for the discussed externality generated by the knowledge creation process.

${ }^{6}$ A large literature stresses that actually the IPR system entails huge administrative and legal costs (see, e.g., Scotchmer [2004] and Cremers et al. [2016], and the references quoted therein). Moreover it has been observed that incumbent patent holders are sometimes dissipating profits for endorsing preemptive strategies or for accumulating costly patent portfolios used as 'bargaining chips' (see, e.g., Gilbert and Newbery [1982], Hall and Ziedonis[2001]). For a model of economic growth à la Romer (1990) that internalizes the enforcement cost of patents see Eicher and García-Peñalosa (2008).
} 
where $\left(\eta^{\prime}\right)^{-1}$ denotes the inverse of the derivative of the probability $\eta$, which clearly exists as $\eta^{\prime}(\cdot)$ is decreasing.

To study the R\&D sector as a whole, note that, as long as the number of identical $R \& D$-firms is $n$, the total amount of resources they use is

$$
J^{*}=n J_{i}^{*}
$$

where $J_{i}^{*}$ is given by (8). Moreover, in equilibrium the whole amount of seeds must also be used, and the firms expectations must turn out to be true, that is:

$$
H(\dot{A}, A)=n \bar{H}_{i}
$$

must hold, where $H(\dot{A}, A)$ represents the whole amount of seeds available in the economy. Since, however, each firm ignores the externalities in terms of seed formation stemming from its own and from the other firms' new knowledge production, it turns out that each firm underestimates the $J$ marginal product, that is, it ignores the (strictly positive) term $\left(\partial / \partial H_{i}\right)\left[\psi H_{i} \eta\left(J_{i} / H_{i}\right)\right]\left(\partial H_{i} / \partial \dot{A}\right)(\partial \dot{A} / \partial J)$ in its decision on $J$. Hence there is an allocative inefficiency as too little $J$ is used. On the other hand, there is no productive inefficiency, since the recombinant process implies that the amount of seeds $H(\dot{A}, A) / n$ adapts to the amount $J_{i}^{*}$ of resources actually employed in production. That is, as long as, e.g., $J_{i}^{*}$ turns out to be a half of the amount a benevolent dictator would choose, also $\bar{H}_{i}$ is a half. Hence, production occurs under constant returns to scale and each firm locates on the same expansion path as under an allocative efficient solution, even on a point nearer to the origin. We can thus focus on a representative firm delivering the whole new knowledge production and using an amount of resource $J^{*}=n J_{i}^{*}$ and $H(\dot{A}, A)=n \bar{H}_{i}$. To describe the equilibrium of the economy as a whole, even if new knowledge $\dot{A}$ is sold in discrete amounts covered by patents, for simplicity we treat the whole industry output, $\dot{A}$, as a continuous function of time.

By plugging $J^{*}$ as in (9) into (1) we get the new knowledge level that the representative firm is willing to supply in our economy, as determined by its input choice:

$$
\dot{A}=H \eta\left[\left(\eta^{\prime}\right)^{-1}(1 / \psi)\right]
$$

In order to explicitly compute $\psi$, we equate the representative $R \& D$-firm's expected revenues to the amount of resources, $G$, paid by the regulator for purchasing the newly created knowledge:

$$
G=\psi \dot{A}
$$

Substituting $\psi$ as in (11) into (10) yields

$$
\dot{A}=H \eta\left[\left(\eta^{\prime}\right)^{-1}(\dot{A} / G)\right],
$$

which, after replacing $H$ as in (2), leads to

$$
\dot{A}=G \eta^{\prime}\left\{\eta^{-1}\left[1 / C_{m}^{\prime}(A)\right]\right\} .
$$

In view of (11), (12) can be rewritten as

$$
\dot{A}=\frac{G}{\psi(A)},
$$


where $\psi$

$$
\psi(A)=\frac{1}{\eta^{\prime}\left\{\eta^{-1}\left[1 / C_{m}^{\prime}(A)\right]\right\}}=\left(\eta^{-1}\right)^{\prime}\left[\frac{1}{C_{m}^{\prime}(A)}\right]
$$

provides an explicit formula for the expected price of knowledge production as a function of the stock of knowledge $A$ paid by the regulator to $R \& D$-firms. As already mentioned earlier, note that the price of knowledge as in (14) is an equilibrium price arising from the matching of knowledge demand (from the government) and supply (by $R \& D$ firms).

The law of motion defined by (13) is directly comparable to that defined by (3) for the first-best economy. Our first result is at the root of the differences between the centralized and the decentralized economy.

\section{Proposition 1}

(i) For any given (finite) stock of knowledge, A, the price of knowledge production for the economy with decentralized RGD production, $\psi(A)$ defined in (14), is always larger than the cost $\varphi(A)$ defined in (4) borne by the social planner in the first-best economy:

$$
\psi(A)>\varphi(A) \quad \text { for all } A<\infty .
$$

(ii) However, when knowledge becomes abundant they converge to the same value:

$$
\lim _{A \rightarrow \infty} \psi(A)=\lim _{A \rightarrow \infty} \varphi(A)=\frac{1}{\eta^{\prime}(0)} .
$$

Proposition 1 states that, while the price of knowledge production in a decentralized R\&D economy is always larger than the cost borne by the social planner in the first-best economy, as the stock of knowledge gets larger and larger, both converge to the same value. For an intuition about this result note that in this economy there is an allocative inefficiency, implying a too small supply, since the $R \& D$-firms, by taking $H$ as given, underestimate the marginal product of factor $J$, and thus use a too small amount of it, while still producing at the unit $\operatorname{cost} \varphi(A)$. Since the regulator's demand has unit elasticity to price, a supply smaller than the efficient one entails a larger equilibrium price. As $H$ becomes abundant, however, the underestimation is fading out and thus the price $\psi(A)$ converges to the unit cost $\varphi(A)$.

Proposition 2 Condition (7) always holds under Assumption A.1; moreover, the wedge between the revenue and the variable cost of the representative $R \& D$-firm turns out to be

$$
[\psi(A)-\varphi(A)] \dot{A}
$$

so that, by Proposition 1 (i), is strictly positive for all $A<\infty$.

Proposition 2 implies that the $R \& D$-firms obtain a positive rent thanks to the externality they enjoy. Thus, the basic unwanted effects of an IPRs system are: i) from the distributive point of view, the private appropriation by the IPR holder of a rent pertaining to a public good, i.e., new knowledge items and ii) a price of new knowledge larger than its marginal social cost, as the positive externalities generated by the new knowledge production translate into a private rent. Proposition 1 (ii) states that these drawbacks tend to fade away in mature economies, where the stock $H$ is at any rate already abundant. Poor countries aiming at starting a growth 
process anew, instead, are likely to find the IPR system more costly. This implies that the IPRs system raises problems not only in terms of efficiency, but also of equity. ${ }^{7}$

In view of Proposition 2, the free entry condition for the $R \& D$ market implies that, after taking into account the total fixed cost for legal expenses, $\mu=n \mu_{i}$, the representative $R \& D$-firm has zero profit,

$$
\pi(A)=[\psi(A)-\varphi(A)] \dot{A}-\mu=0,
$$

that is, the market is in equilibrium and no further entry occurs as long as the firms' rent is fully "burned down" to finance the preventive fixed cost $\mu$ aimed at discouraging legal challenges. ${ }^{8}$

We can thus summarize the main features of the $R \& D$ market equilibrium as follows:

(i) supply of new knowledge is made by $n$ identical firms who maximize profit by choosing the amount of resources $J$ for processing an amount $H / n$ of freely available seeds that they take as exogenously given;

(ii) as each firm ignores the external effects of new knowledge production on the seeds' availability, it employs too few resources $J$, whose amount thus results scaled down with respect to an allocative efficient solution;

(iii) thanks to the recombinant process, also the number of seeds used by each firm turns out to be scaled down by the same factor and thus production occurs under constant returns to scale, on the same expansion path that would be chosen under an efficient solution;

(iv) the allocative inefficiency implies an undersupply of knowledge and, as demand has unitary elasticity, the new knowledge price is larger than that (equal to the cost) one can account for under a benevolent planner's solution;

(v) $R \& D$ firms, which produce at the same cost as under the benevolent planner's solution, enjoy a rent which, however, in equilibrium under free entry is fully dissipated into a fixed cost to prevent challenges to the IPRs.

\subsection{Government}

In our stylized framework the government levies lump-sum taxes on households in order to buy and then to distribute for free across the economy newly produced ideas, maintaining a balanced budget at any point in time. Additionally, the government can correct the market failure arising in the innovative sector and restore optimality. The government basically resorts to a tax-subsidy scheme in order to improve efficiency; as the problem is that the price of the private provision of knowledge via IPRs is larger than the social cost, the corrective intervention should consist in a subsidy to the knowledge production, financed through a non distortionary tax on the rent of the $R \& D$-firms. ${ }^{9}$

\footnotetext{
${ }^{7}$ Ramello (2008) notes that, because of indivisibility characterizing the knowledge production, there is a unique link between efficiency and equity, and the intensity of appropriation via IPRs can thus negatively affect both at the same time.

${ }^{8}$ For the sake of simplicity we model the zero profit condition as deterministic, while the model's results would not change if we assume that the zero profit condition holds at aggregate level, under idiosyncratic uncertainty about the occurrence and the result of disputes.

${ }^{9}$ Note that our economy presents two inefficiencies (a public good and an externality), so that two policy instruments (the tax on households and the tax-subsidy mechanism on $R \& D$-firms) are needed in order to restore optimality as in standard theory of economic policy (Tinbergen rule). The peculiarity of such inefficiencies is related to the double nature of knowledge, as input (seeds generate an externality) and output (the recombinant process generates new knowledge, a public good).
} 
More specifically, as long as the government aims at implementing the efficient knowledge production that would arise under centralization, $\dot{A}=J / \varphi(A)$ as defined in (3), it should increase its own demand - with respect to the level that the latter would take given $G<J$, i.e., the $\mathrm{R} \& \mathrm{D}$ financing under decentralization according to $(11)$ and the price $\psi(A)$ - so that

$$
\frac{G+d}{\psi(A)}=\frac{J}{\varphi(A)}=\dot{A}
$$

where $d$ is the subsidy needed to correct the market failure. Assuming that the government can levy taxes directly on $R \& D$-firms' rents at a rate $0 \leq \tau \leq 1$, according to (17) we can set ${ }^{10} d=\tau[\psi(A)-\varphi(A)] \dot{A}$, so that, by (18), $G+d=G+\tau[\psi(A)-\varphi(A)] \dot{A}=\psi(A) \dot{A}$, which immediately yields the law of motion of knowledge that will specify our version of the recombinant growth model:

$$
\dot{A}=\frac{G}{\phi_{\tau}(A)},
$$

where

$$
\phi_{\tau}(A)=\tau \varphi(A)+(1-\tau) \psi(A),
$$

with $\varphi(A)$ and $\psi(A)$ defined in (4) and (14) respectively.

If the government, by setting $\tau=1$, were able to fully expropriate the $R \& D$-firms' rents, the same first-best knowledge growth path observed under centralization would be reached through a redistributive process based on the tax-subsidy scheme. While in principle this would fully fix the efficiency problem, such implementation is generally not possible because it requires an exact assessment and expropriation of the firm's rent, a task generally deemed outside the reach of the government in a decentralized economy, where asymmetry of information characterizes the relationship between the government and the private sector. On the other hand, $\tau=0$ implies fully decentralized, inefficient knowledge production where, however, the rent appropriation by the patent holder is maximal.

The main focus of this paper is on partial implementations of such a scheme. Specifically, we aim at studying how the economy as a whole reacts to different values of the parameter $0 \leq$ $\tau \leq 1$, which is assumed to be constant over time, in terms of long-run equilibria and transition time-path trajectories of the main variables involved, like stock of knowledge, output and consumption, their growth rates and welfare. Since different tax policies (different $\tau$ ) are associated with different degrees of private rent appropriability in the research market (different $1-\tau)$, by studying the impact on growth and welfare of different tax policies we also analyze their relationship with the degree of rent appropriability. In the proceeding of the paper we will refer to different tax regimes (or alternatively different appropriability regimes) rather than different IPRs policy regimes, in order to stress that the notion of policy and the type of IPRs system we are considering are substantially different from those traditionally studied in literature.

\section{Equilibria and Endogenous Growth}

Given the policy parameter $0 \leq \tau \leq 1$, in general equilibrium all markets clear and our economy can be summarized via a welfare maximization problem where the social planner chooses the optimal amount $G$ to be employed for buying new knowledge and the optimal level

\footnotetext{
${ }^{10}$ Note that, in order to be non distortionary, the tax should be levied as a share of pure rents. As long as the latter are cut by taxation, the fixed cost born by firms, aimed at discouraging those who might contend their IPRs - which previously where assumed to exhaust profits - can be cut accordingly.
} 
of consumption $c$ in order to maximize the representative household lifetime utility, subject to the resource constraint

$$
c(t)+G(t)+X(t)=Y(t),
$$

given the law of motion of knowledge in (19) and the initial condition $A(0)=A_{0}$. Recalling that the intermediate good $X$ is priced at the numeraire and that $f(\cdot)$ in $(5)$ has invertible first order derivative, the planner first maximizes net output, $Y(t)-X(t)=$ $A(t) f[X(t) / A(t)]-X(t)$, with respect to $X(t)$, obtaining the optimal amount of intermediate good $X^{*}(t)=\left(f^{\prime}\right)^{-1}(1) A(t)$ which, after replacing it into (21), yields the net output $Y(t)-X(t)=A(t) f\left[\left(f^{\prime}\right)^{-1}(1)\right]-\left(f^{\prime}\right)^{-1}(1) A(t)$, that is,

$$
Y(t)-X(t)=\delta A(t), \quad \text { with } \delta \equiv f\left[\left(f^{\prime}\right)^{-1}(1)\right]-\left(f^{\prime}\right)^{-1}(1) .
$$

In other words, the optimal net output turns out to be linear in $A$ and the ratio $X^{*} / A$ is constant through time: $X^{*} / A \equiv\left(f^{\prime}\right)^{-1}(1)$.

Therefore, the resource constraint $(21)$ becomes $G(t)=\delta A(t)-c(t)$, which, in view of (19), allows to state the social planner problem as

$$
\begin{gathered}
V\left(A_{0}\right)=\max _{[c(t)]_{t=0}^{\infty}} \int_{0}^{\infty} e^{-\rho t} u[c(t)] \mathrm{dt} \\
\text { subject to }\left\{\begin{array}{l}
\dot{A}(t)=\frac{\delta A(t)-c(t)}{\phi_{\tau}[A(t)]} \\
A(0)=A_{0}
\end{array}\right.
\end{gathered}
$$

plus the usual non-negativity constraints. The stock of knowledge $A(t)$ is the state variable and consumption $c(t)$ is the control. Denoting by $\lambda(t)$ the costate variable associated with the unique dynamic constraint in (24) and dropping the time argument for simplicity, the current-value Hamiltonian associated to $(23)$ is

$$
H(A, c, \lambda)=u(c)+\lambda \frac{\delta A(t)-c(t)}{\phi_{\tau}[A(t)]} .
$$

Unfortunately, the Hamiltonian in (25) turns out not to be concave and multiple equilibria can arise. Specifically, two regimes can occur: 1) a stagnating equilibrium in which all net output $Y(t)-X(t)=\delta A(t)$ is consumed at each instant $t$, defined by a constant consumption $\bar{c}(t) \equiv \delta A_{0}$, so that no investment is made in piling up new knowledge, $G(t) \equiv 0$, corresponding to zero growth, $\dot{A}(t) / A(t)=\dot{Y}(t) / Y(t)=\dot{c}(t) / c(t) \equiv 0$; and 2) an equilibrium envisaging sustained growth-i.e. $\dot{A}(t) / A(t)>0, \dot{Y}(t) / Y(t)>0, \dot{c}(t) / c(t)>0$-possibly converging to an asymptotic balanced growth path (ABGP). To focus on the latter we compute necessary conditions on (25):

$$
\begin{gathered}
u^{\prime}(c)=\frac{\lambda}{\phi_{\tau}(A)} \\
\dot{\lambda}=\rho \lambda-\lambda \frac{\delta \phi_{\tau}(A)-(\delta A-c) \phi_{\tau}^{\prime}(A)}{\left[\phi_{\tau}(A)\right]^{2}} \\
\lim _{t \rightarrow+\infty} e^{-\rho t} \lambda(t) A(t)=0,
\end{gathered}
$$

where (28) is the transversality condition. Differentiating (26) with respect to time we get

$$
\frac{\dot{\lambda}}{\lambda}=\frac{\phi_{\tau}^{\prime}(A) \dot{A}}{\phi_{\tau}(A)}-\varepsilon_{u}(c) \frac{\dot{c}}{c},
$$


where $\varepsilon_{u}(c)=-u^{\prime}(c) /\left[u^{\prime \prime}(c) c\right]$ is the inverse of the intertemporal elasticity of substitution. Coupling (29) with (27), using the dynamic constraint in (24) and rearranging terms we obtain the following instantaneous consumption growth rate:

$$
\gamma(t)=\frac{\dot{c}(t)}{c(t)}=\frac{1}{\varepsilon_{u}[c(t)]}\left\{\frac{\delta}{\phi_{\tau}[A(t)]}-\rho\right\} .
$$

As $\phi_{\tau}[A(t)]$ changes over time (possibly, $\varepsilon_{u}[c(t)]$ as well), (30) implies the presence of transition dynamics. In the next proposition we focus on an ABGP equilibrium. To this aim, note that, by Proposition 1(ii) and the definition of knowledge unit cost in (20), the latter converges to a constant value as $A \rightarrow+\infty$; therefore, for $A$ becoming larger and larger as time elapses, according to (16) $\lim _{t \rightarrow+\infty} \phi_{\tau}[A(t)]=1 / \eta^{\prime}(0)$, where $\eta^{\prime}(0)$ denotes the slope of the probability function $\eta(\cdot)$ when zero resources are employed for matching ideas in the recombinant process (see Assumption A.1), that is, the efficiency of the first dollar spent in R\&D activities. Thus, if we also assume that asymptotically the intertemporal elasticity of substitution becomes constant, $\lim _{t \rightarrow+\infty}\left[1 / \varepsilon_{u}(c)\right]=1 / \sigma, \sigma>0$, we immediately obtain the following characterization.

Proposition 3 Assume that $\lim _{t \rightarrow+\infty}\left[1 / \varepsilon_{u}(c)\right]=1 / \sigma, \sigma>0$. Then, for any tax policy parameter value $0 \leq \tau \leq 1$, if the initial stock of knowledge, $A_{0}$, is sufficiently large, that is, $A_{0} \geq A_{\tau}^{s k}$, where the value $A_{\tau}^{\text {sk }}$ depends on the tax parameter $\tau$, and the following conditions are satisfied,

$$
(1-\sigma) \delta \eta^{\prime}(0)<\rho<\delta \eta^{\prime}(0)
$$

then

(i) the economy admits a unique ABGP along which knowledge, output, and consumption all grow at the same asymptotic growth rate given by

$$
\gamma^{*}=\frac{\dot{c}}{c}=\frac{\dot{A}}{A}=\frac{\dot{Y}}{Y}=\frac{\delta \eta^{\prime}(0)-\rho}{\sigma} ;
$$

(ii) the ABGP equilibrium characterized by (32) is Pareto optimal;

(iii) the optimal consumption along the ABGP equilibrium is a constant fraction (or a multiple) of the stock of knowledge; that is, asymptotically the optimal policy is linear in A:

$$
c^{*}(A)=\frac{1}{\sigma}\left[(\sigma-1) \delta+\frac{\rho}{\eta^{\prime}(0)}\right] A
$$

Provided that some technical conditions are met, Proposition 3 establishes that the economy in the long-run converges to an ABGP along which knowledge, output, and consumption all grow at the same constant rate given by (32), and with constant income share devoted to consumption according to (33). ${ }^{11}$ In order for the ABGP to be well defined we need that the initial stock of ideas, $A_{0}$, is large enough, ${ }^{12}$ the asymptotic inverse elasticity of substitution, $\sigma$, is large enough, and households are patient enough with respect to the technology employed in final production, $\delta$, jointly with the efficiency of the Weitzman recombinant mechanism generating new knowledge when little resources are employed in matching hybrid ideas, $\eta^{\prime}(0)$.

\footnotetext{
${ }^{11}$ See Palivos et al. (1997) for the existence of BGP and ABGP equilibria.

${ }^{12}$ The initial amount of knowledge $A_{0}$ behaves like a Skiba-type point, $A_{\tau}^{s k}$, selecting between the two types of equilibria mentioned above: if $A_{\tau}^{s k}<A_{\tau}^{s k}$ the economy is doomed to stagnation forever, while when $A_{0} \geq A_{\tau}^{s k}$ the economy asymptotically converges to sustained growth at the constant rate given by (32).
} 
Remark 1 Along the ABGP, the condition for growth (31), the long-run economic growth rate in (32) and the optimal policy (33) turn out to be completely independent of the tax policy parameter $\tau$; only the eventual Skiba-type point $A_{\tau}^{\text {sk }}$ depends on $\tau$. Therefore, the growth behavior of the economy in the long run is not affected by whether new knowledge is being produced in a centralized manner or under decentralization by means of IPRs (with or without corrective tax-subsidy interventions), reaching always a first-best equilibrium.

The above Remark 1 clearly states that the ABGP outcome is completely independent upon the adopted tax (and thus appropriability) regime. However, according to (30), the determined degree of tax intervention (thus the determined degree of rent appropriability) does affect the transitional dynamics and therefore the level of social welfare. In order to understand how welfare is related to different tax regimes, we need to analyze how the whole transitional dynamic path is affected by the tax parameter.

\section{Model Specification and Transition Dynamics}

Following Privileggi $(2010 ; 2011 ; 2015)$, in the sequel we shall assume that only pairs of ideas can be matched and that the probability of success is described by a hyperbolic function.

A. $2 m=2$ and the success probability function is given by

$$
\eta(x)=\beta x /(\beta x+1), \quad \beta>0 .
$$

It is immediately seen that $\eta(\cdot)$ as defined in (34) satisfies Assumption A.1 with $\eta^{\prime}(0)=\beta$ and $\eta^{\prime \prime}(0)=-2 \beta^{2}$. Parameter $\beta$ provides a measure of the 'degree of efficiency' of the Weitzman matching process: the larger $\beta$ the higher the probability of obtaining a new successful idea out of each (pairwise) matching of seed ideas. For $m=2, C_{2}^{\prime}(A)=(2 A-1) / 2$ and from (34) we get $\eta^{-1}(x)=x /[\beta(1-x)]$; using these into (4) and (14) we get

$$
\begin{aligned}
& \varphi(A)=\frac{1}{\beta}\left(\frac{2 A-1}{2 A-3}\right)=\frac{1}{\beta}\left(1+\frac{2}{2 A-3}\right) \\
& \psi(A)=\frac{1}{\beta}\left(\frac{2 A-1}{2 A-3}\right)^{2}=\frac{1}{\beta}\left(1+\frac{2}{2 A-3}\right)^{2},
\end{aligned}
$$

which, when substituted in (20), yield the following expression for the unit cost of knowledge production:

$$
\phi_{\tau}(A)=\varphi(A)[(1-\tau) \beta \varphi(A)+\tau]=\frac{(2 A-1)(2 A-2 \tau-1)}{\beta(2 A-3)^{2}},
$$

defined for $A>3 / 2$ and $0 \leq \tau \leq 1$. Note that, thanks to (36), the first equality in (37) defines $\phi_{\tau}(A)$ as a function of the unit cost $\varphi(A)$ introduced in (4). It is immediately seen that, for each given $\tau, \phi_{\tau}(A)$ is decreasing in $A$, while Proposition 1 implies that $\psi(A) \geq \phi_{\tau}(A)>\varphi(A)$ for all $A>3 / 2$ and all $0 \leq \tau \leq 1$, and $\lim _{A \rightarrow \infty} \phi_{\tau}(A)=\lim _{A \rightarrow \infty} \varphi(A)=1 / \eta^{\prime}(0)=1 / \beta>0$.

A. 3 Output is produced according to a Cobb-Douglas technology:

$$
y(t)=F[X(t), A(t)]=\theta[X(t)]^{\alpha}[A(t)]^{1-\alpha}, \quad \theta>0,0<\alpha<1,
$$

depending on an intermediate good, $X(t)$, and knowledge-augmented labor, $A(t) L(t)$, for $L(t) \equiv 1$. 
In assuming decreasing returns to each factor taken alone we follow Weitzman (1998) and Tsur and Zemel (2007), thus departing from the standard approach which excludes decreasing returns to scale to knowledge. ${ }^{13}$ Weitzman (1998, p. 344) notes that knowledge used as input in the production of final goods should be measured in units of "effective augmentation" of the output. The quantification of the available stock of knowledge thus might be different from that relevant from the point of view of the recombination process. Making the assumption of decreasing returns to scale to knowledge in production while using a unique scale of measurement of knowledge is thus equivalent to assuming that the suitable scale for the use in production should keep into account some loss of effectiveness, due, e.g., to coordination difficulties, partial substitution of previously used results, etc.

Each output producing $F$-firm maximizes instantaneous profit by purchasing the intermediate good $X$ and hiring labor $L$ from the households, taking as given the price of $X$, which equals 1 as it is the numeraire, the labor wage $w$ and the stock of knowledge $A$. Since $F$-firms act competitively, their profits are zero.

Aggregate output can be rewritten as

$$
y(t)=\theta[X(t)]^{\alpha}[A(t)]^{1-\alpha}=A(t) \theta\left[\frac{X(t)}{A(t)}\right]^{\alpha},
$$

that is, in view of $(5), f(x)=\theta x^{\alpha}$. Using (39) it is immediately seen that $\left(f^{\prime}\right)^{-1}(x)=$ $(\theta \alpha)^{1 /(1-\alpha)} x^{1 /(\alpha-1)}$, so that $\left(f^{\prime}\right)^{-1}(1)=(\theta \alpha)^{1 /(1-\alpha)} \equiv X(t) / A(t)$ for all $t \geq 0$, and

$$
\delta \equiv f\left[\left(f^{\prime}\right)^{-1}(1)\right]-\left(f^{\prime}\right)^{-1}(1)=\theta(\theta \alpha)^{\alpha /(1-\alpha)}-(\theta \alpha)^{1 /(1-\alpha)}=(1-\alpha) \theta^{1 /(1-\alpha)} \alpha^{\alpha /(1-\alpha)},
$$

which, according to (22), yields the optimal net aggregate output

$$
Y(t)-X(t)=\delta A(t)=(1-\alpha) \theta^{1 /(1-\alpha)} \alpha^{\alpha /(1-\alpha)} A(t) .
$$

A. 4 The instantaneous utility function is CIES,

$$
u(c)=\frac{c^{1-\sigma}-1}{1-\sigma}, \quad \sigma>0 .
$$

As in Tsur and Zemel (2007), Privileggi (2010; 2011; 2015) and Privileggi and Marsiglio (2014), under Assumptions A.2-4 two optimal regimes are possible:

1. zero R\&D, $G(t)=\delta A(t)-c(t) \equiv 0$, implying a constant optimal consumption level given by $\bar{c}(t) \equiv \delta A_{0}$ which immediately puts the economy on the steady state $\left(A_{0}, \delta A_{0}\right)=$ $\left(A_{0},(1-\alpha) \theta^{1 /(1-\alpha)} \alpha^{\alpha /(1-\alpha)} A_{0}\right)$ in the $(A, c)$ space, and yields a social welfare given by

$$
\begin{aligned}
\bar{V}\left(A_{0}\right) & =\int_{0}^{\infty} \frac{\bar{c}(t)^{1-\sigma}-1}{1-\sigma} e^{-\rho t} \mathrm{dt}=\frac{\left(\delta A_{0}\right)^{1-\sigma}-1}{1-\sigma} \int_{0}^{\infty} e^{-\rho t} \mathrm{dt} \\
& =\frac{\left[(1-\alpha) \theta^{1 /(1-\alpha)} \alpha^{\alpha /(1-\alpha)} A_{0}\right]^{1-\sigma}-1}{(1-\sigma) \rho}
\end{aligned}
$$

\footnotetext{
${ }^{13}$ The standard argument relies on the concept of replicability: to double the copies of a design you may need to double all the factors but you do not need to double the design. The idea is that once the right to use a certain blueprint or idea has been obtained (through purchase or for free), such a blueprint can be used in all the production sites.
} 
2. under the assumptions of Proposition 3, namely $(1-\sigma) \beta(1-\alpha) \theta^{1 /(1-\alpha)} \alpha^{\alpha /(1-\alpha)}<\rho<$ $\beta(1-\alpha) \theta^{1 /(1-\alpha)} \alpha^{\alpha /(1-\alpha)}$, an optimal path satisfying the dynamic constraint in (24) and the equation (30):

$$
\left\{\begin{array}{l}
\dot{A}=\frac{\left[(1-\alpha) \theta^{1 /(1-\alpha)} \alpha^{\alpha /(1-\alpha)} A-c\right] \beta(2 A-3)^{2}}{(2 A-1)(2 A-2 \tau-1)} \\
\dot{c}=\frac{1}{\sigma}\left[\frac{(1-\alpha) \theta^{1 /(1-\alpha)} \alpha^{\alpha /(1-\alpha)} \beta(2 A-3)^{2}}{(2 A-1)(2 A-2 \tau-1)}-\rho\right] c
\end{array}\right.
$$

envisaging growth as time elapses and eventually leading to an ABGP characterized by the (common, constant) growth rate (32) independent of parameter $\tau$ :

$$
\gamma^{*}=\frac{\dot{c}}{c}=\frac{\dot{A}}{A}=\frac{\dot{Y}}{Y}=\frac{\delta \beta-\rho}{\sigma} .
$$

In order to study how a given economy reacts to different tax policies (different values of $0 \leq \tau \leq 1$, assumed to be constant over time) we perform comparative dynamics by changing the value of parameter $\tau$ while keeping constant all other parameters' values. Specifically, we aim at 1) studying if and how the Skiba-type point $A_{\tau}^{s k}$ changes for different $\tau$-values and 2) comparing social welfare under different tax regimes.

Because the system of ODEs in (42) cannot be solved in closed form, in order to evaluate social welfare in the latter scenario described above we rely on a numerical approach aimed at estimating the whole optimal time-path trajectories of the stock of knowledge, $A(t)$, and the optimal consumption, that in the sequel will be denoted by $\tilde{c}(t)$ to emphasize that it is the (approximated) solution of (42). The results of such simulation show that it is not obvious at all to make inferences on social welfare only by looking at time-path trajectories, as, due to time discounting, higher values of $\tau$, which squeeze consumption in early stages to boost it later thanks to faster knowledge accumulation, may well fail to deliver higher levels of welfare. Hence, in order to assess the role of the tax policy $\tau$ a reliable estimate of social welfare is needed, a task which the whole next section is dedicated to.

\section{$5 \quad$ Simulations and Welfare Analysis}

The technical details of the whole numerical analysis preformed in this section, based on a time elimination approach (Mulligan and Sala-i-Martin [1991]) are reported in Appendix B. We assume the following values for the fundamental parameters in our economy, which are common in the macroeconomic literature (see, e.g., Mulligan and Sala-i-Martin [1993]):

$$
\rho=0.04, \quad \alpha=0.33, \quad \theta=1, \quad \sigma=2, \quad \beta=0.20 .
$$

Our goal is to perform comparative dynamics analysis under eleven different values of the policy parameter, $0 \leq \tau \leq 1$, which are assumed to be constant over time. Specifically, we consider

$$
\tau=0,0.1,0.2, \ldots, 0.9,1
$$

It is worth to remind that in terms of rent appropriability by $R \& D$-firms via IPRs, $\tau=0$ supplies to the right holder the maximal degree, while the other values indicate progressively decreasing appropriability levels, reaching a minimum when $\tau=1$, corresponding to the firstbest solution as in this case new knowledge is being supplied exactly at its cost $\varphi(A)$ defined 
in (4). According to Proposition 3, the long-run balanced growth occurs at the same constant growth rate given by $(32):{ }^{14}$

$$
\gamma^{*}=\frac{\dot{A}}{A}=\frac{\dot{y}}{y}=\frac{\dot{c}}{c}=\frac{\delta \beta-\rho}{\sigma}=0.0188
$$

Figure 1 shows in the $(A, c)$ space the linear asymptotic policy defined by $c^{*}(A)=(1 / \sigma)[(\sigma-1) \delta+\rho / \beta] A=0.2940 A$ (the line on the bottom of the graph) and all the eleven transitory optimal policies $\tilde{c}_{\tau}(A)$ approaching $c^{*}(A)$ from above, for $\tau=0,0.1, \ldots, 1$, where the initial stock of knowledge $A_{0}=4.0107$, common to all policy parameters $\tau$, is the minimum value that allows the numerical method described in Appendix B to work. It is clearly seen that all such policies are monotonically decreasing in $\tau$ : specifically, for each fixed $A$ value, smaller optimal consumption values $\tilde{c}_{\tau}(A)$ correspond to larger $\tau$-values, with $\tilde{c}_{\tau=0}(A)$ being the graph on top and $\tilde{c}_{\tau=1}(A)$ being the graph on the bottom of the nonlinear curves, above the linear asymptotic policy $c^{*}(A)$. In the figure only a small range, $[4.0107,6]$, of values of knowledge close to the lower bound needed to support growth is emphasized, where differences among optimal policies are more notable; for larger values of $A$ the monotonicity in $\tau$ is preserved, with all policies graphs slowly converging toward the linear asymptotic policy $c^{*}(A)$ from above.

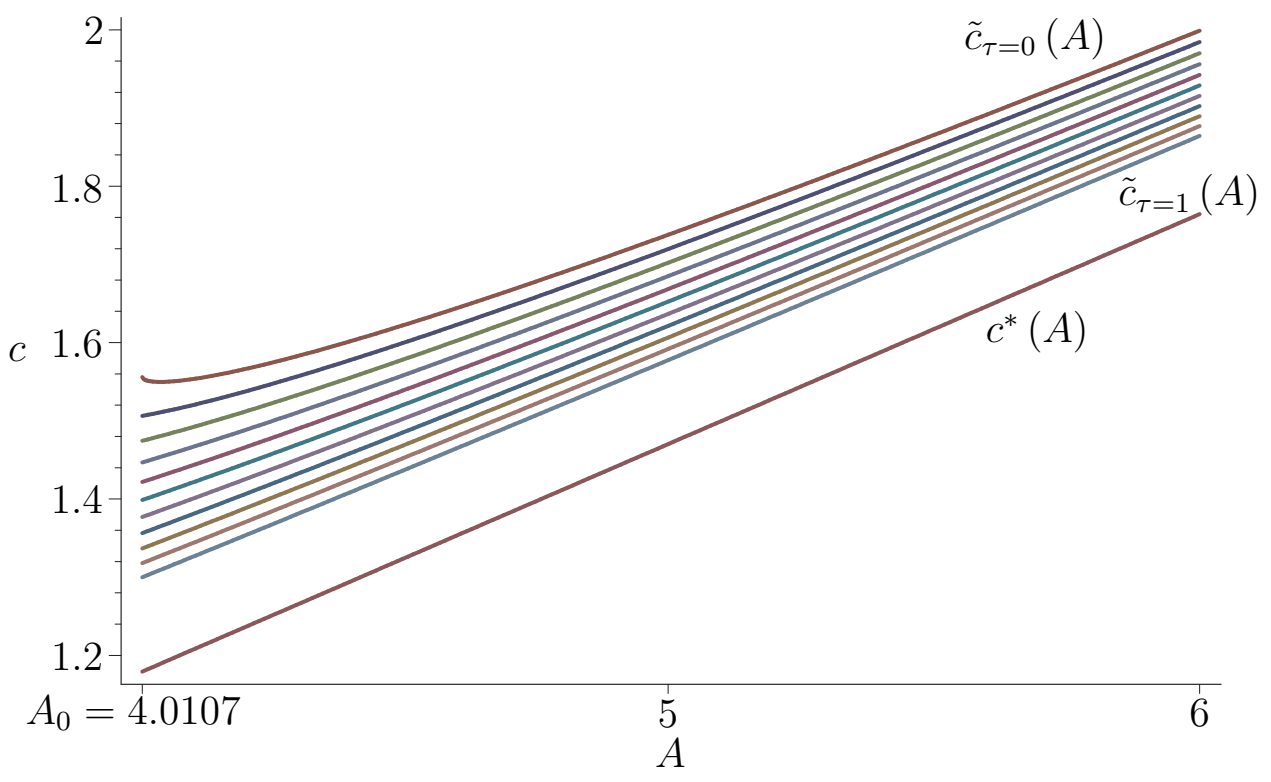

FiguRE 1: the linear asymptotic policy $c^{*}(A)$ and all eleven transitory policies under the tax-subsidy regimes $\tau=0,0.1, \ldots, 1$.

Figure 2(a) plots the whole time-path trajectories of $A(t)$ which confirm their monotonicity property with respect to $\tau$ : larger $\tau$-values imply a faster accumulation of knowledge in time. Similarly, Figure 2(b) clearly shows that the total output dynamics are just a (constant) fraction of the $A(t)$ dynamics. Figures 2(c) and 2(d) report the optimal consumption time-path trajectories: Figure 2(c) shows that, for $t$ sufficiently large, the consumption time-path trajectories follow a pattern similar to that of the stock of knowledge $A(t)$, being larger at the same instant $t$ for larger $\tau$-values. However, in early instants the opposite occurs: larger $\tau$-values are associated to smaller consumption levels, as it is evident in Figure 2(d) for $t<30$.

\footnotetext{
${ }^{14}$ Recall that, from Assumption A.2, $\eta^{\prime}(0)=\beta$. Note also that the parameter values we have chosen imply a growth rate of around $1.88 \%$ in (45), which is very close to that in Acemoglu and Akcigit (2012).
} 


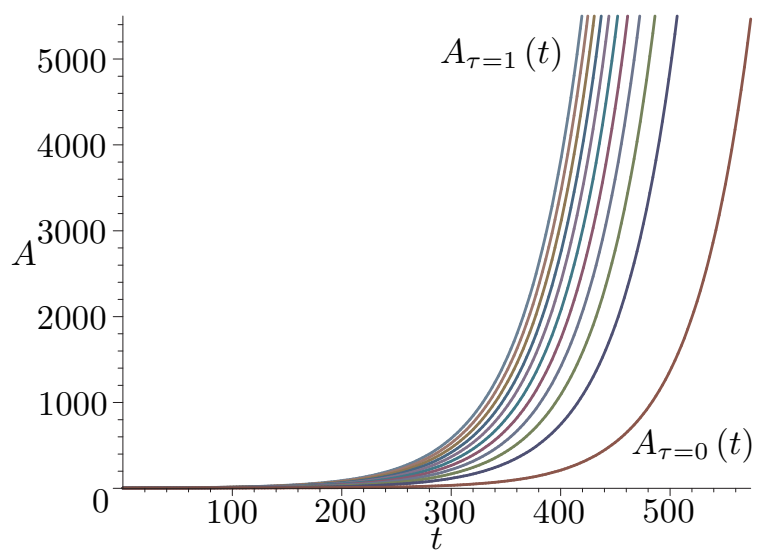

(a)

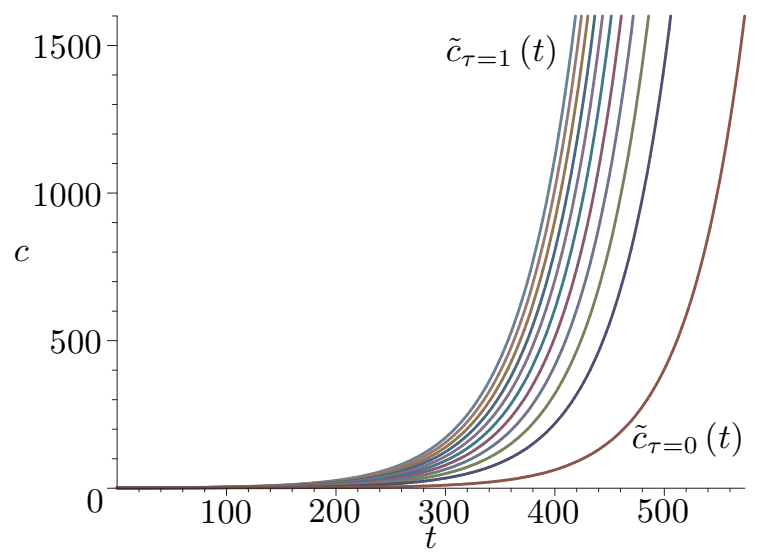

(c)

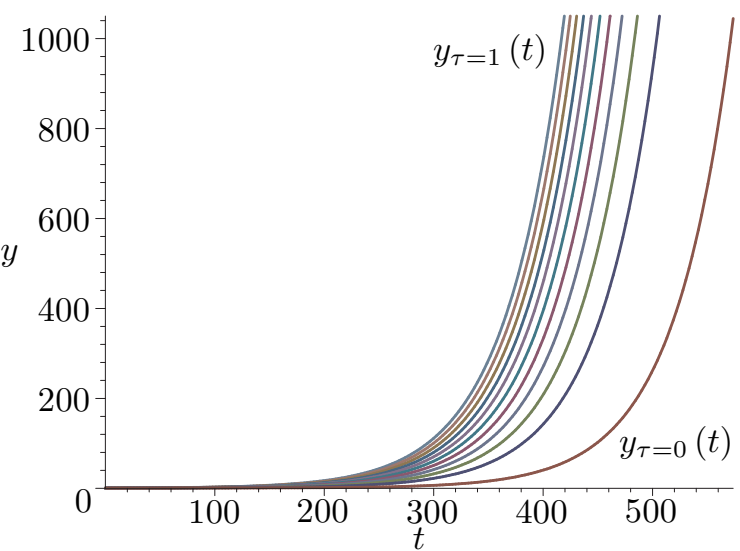

(b)

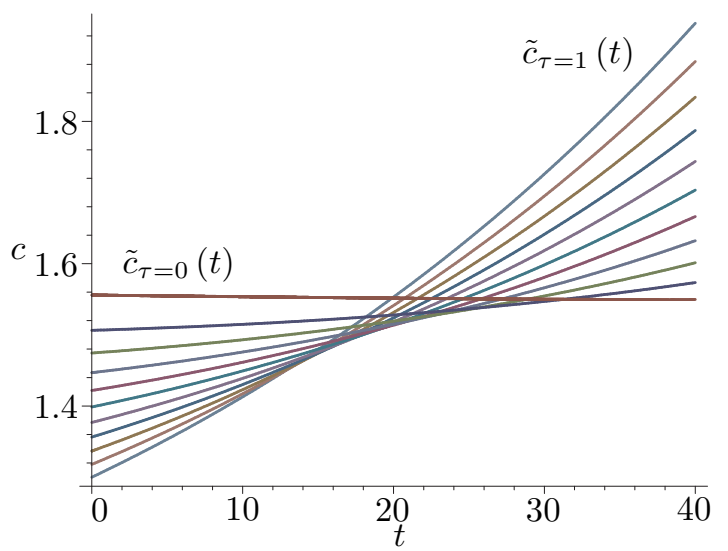

(d)

Figure 2: optimal time-path trajectories, (a) $A(t)$, (b) $y(t)$, (c) $\tilde{c}(t)$, and (d) again $\tilde{c}(t)$ at earlier instants, under the eleven tax-subsidy regimes, $\tau=0,0.1, \ldots, 1$.

From Figure 2(d) it is not clear what the effect of the different tax policies on welfare may be, as in early times larger $\tau$-values envisage smaller consumption, only to catch up and rapidly overcome later on. As a matter of fact, trajectories determined by larger $\tau$-values may fail to deliver a welfare higher than that yielded under smaller $\tau$-values regimes if discounting assigns less weight to later consumption than that assigned to early consumption. Figures $3(\mathrm{a})$ and $3(\mathrm{~b})$ clear any doubt on this by showing the social welfare $\bar{V}(A)$ correspondent to a stagnating economy and the social welfare $V_{\tau}(A)$ for $\tau=0, \tau=0.5$ and $\tau=1$ as functions of any initial stock of knowledge $A \geq A_{0}$, where the latter is being calculated using formula (55) in Appendix B. Especially in Figure 3(b), where a shorter range of $A$ values close to the lower bound $A_{0}=4.0107$ is considered, it is apparent that once again a monotonicity pattern with respect to $\tau$ is confirmed: for all $A \geq 4.0107$ on one hand all $\tau$ regimes (including $\tau=0$ ) characterizing convergence toward sustained growth dominate the stagnating economy represented by $\bar{V}(A)$, while on the other hand larger $\tau$-values generate larger welfare values for each initial knowledge stock $A$. As the graphs of all social welfare functions in the sustained growth scenario lie quite close to each other, in Figures 3(a) and 3(b) we plot only three of them, corresponding to $\tau=0, \tau=0.5$ and $\tau=1$; however this result is robust, as it can be checked that $\tau_{j}>\tau_{i}$ implies $V_{\tau_{i}}(A)>V_{\tau_{j}}(A)$ for any pair $\tau_{i}, \tau_{j}$ such that $\tau_{i}>\tau_{j}$ and for all $A \geq 4.0107$. More specifically, Table 1 reports the welfare values in stagnation and in the eleven $\tau$-regimes considered at the initial common knowledge stock $A_{0}=4.0107$. 


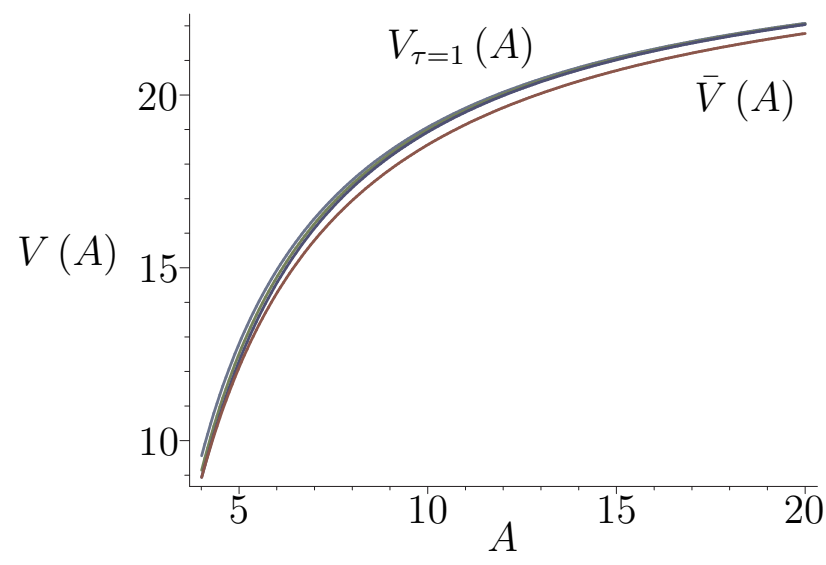

(a)

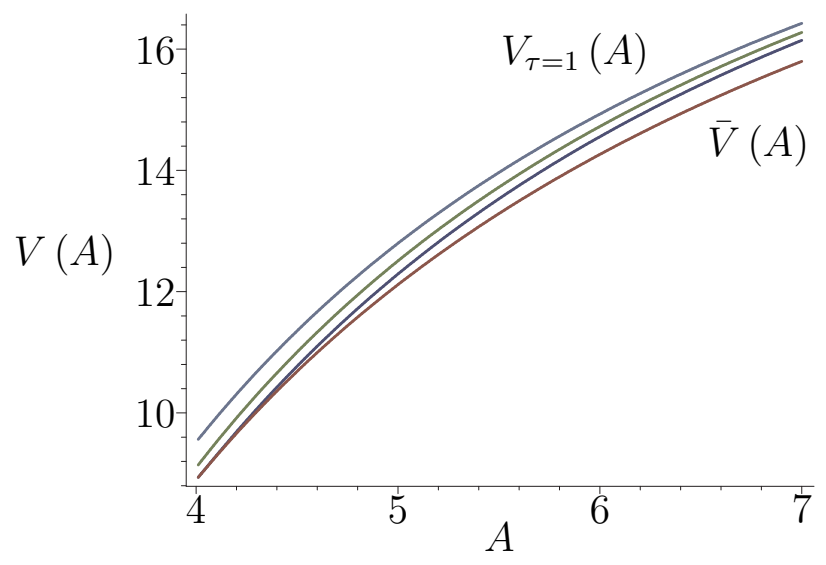

(b)

Figure 3: (a) value functions estimations, $\bar{V}(A)$ in stagnation and $V_{\tau}(A)$ toward sustained growth along the ABGP, for $\tau=0, \tau=0.5$ and $\tau=1$, of problem (23) for the parameters' values in (43), (b) same value functions for values of $A$ closer to $A_{0}=4.0107$.

\begin{tabular}{|c|c|c|}
\hline$\tau$ & Toward & Social welfare \\
\hline 0 & stagnation & 8.938175 \\
0 & ABGP & 8.938178 \\
0.1 & ABGP & 8.955999 \\
0.2 & ABGP & 8.987932 \\
0.3 & ABGP & 9.030416 \\
0.4 & ABGP & 9.082202 \\
0.5 & ABGP & 9.142614 \\
0.6 & ABGP & 9.211234 \\
0.7 & ABGP & 9.287792 \\
0.8 & ABGP & 9.372105 \\
0.9 & ABGP & 9.464056 \\
1 & ABGP & 9.563570 \\
\hline
\end{tabular}

TABLE 1: social welfare in a stagnating economy and in all tax regimes, $\tau=0,0.1, \ldots, 1$, toward sustained growth for the initial knowledge endowment $A_{0}=4.0107$.

Note that in the first two rows of Table 1 the social welfare of a purely decentralized economy $(\tau=0)$ choosing to live forever in stagnation turns out to be very close to that yielded by the same economy when the social planner opts for sustained growth eventually reaching the ABGP. This suggests that the lack of a corrective public intervention risks of undermining the incentives to start the recombinant process.

An even more extreme threat against growth would occur at a Skiba point, i.e., if there was an original endowment of knowledge such that economies starting below it would reach a larger welfare by stagnating than by accumulating further knowledge and growing. To study this case we performed several simulations for different parameters' values. We found quite hard to obtain a scenario in which the social welfare in a stagnating economy dominates the social welfare toward the ABGP, and succeeded only by imposing unrealistic parameter values. ${ }^{15}$ We

\footnotetext{
${ }^{15}$ One of such cases, e.g., requires a very large discount rate $\rho=0.1$, joint with $\alpha=0.5, \theta=1, \sigma=2$, and $\beta=0.60$, corresponding to a long-run asymptotic growth rate $\gamma^{*}=0.025$; in this scenario, starting from an initial stock of knowledge $A_{0}=5.949$, the welfare yielded by stagnation (equal to 3.276180872 ) overcomes
} 
cannot, however, rule out the possibility that a Skiba point does exist also in the more realistic cases we considered, as it might have failed to turn out since out of reach of the numerical algorithm described in Appendix B; this is possible if the Skiba point occurred at values very close to the lower bound for the initial knowledge endowment implied by our model specification. However, since the lower bound in our simulations is not demanding (the recombinant process can start at a very small original knowledge endowment, i.e., at slightly more than 4 successful ideas) such a Skiba point, if any, seems unlikely to preclude growth in most circumstances.

To conclude, the approximated values found through the whole procedure discussed so far lead to the following results:

(i) under all tax regimes, $\tau=0,0.1, \ldots, 1$, the economy grows along the ABGP in the long-run, that is, for the parameters' values chosen in (43) stagnation is always ruled out;

(ii) social welfare is strictly increasing with respect to positive increments of the tax parameter $\tau$-corresponding to progressively stricter tax regimes, i.e., progressively weaker appropriability regimes.

\section{Concluding Remarks}

While thus far the literature has largely recognized that government intervention is needed to fix the inefficiencies deriving from the IPRs system, it has mainly focused on the appropriability of knowledge considering the rights' structural features (e.g., patent breadth or length; see Acemoglu and Akcigit [2012], and the literature quoted therein). We contend here that the focus should be shifted to the rents which the IPRs system gives rise to, and to the corrective role that taxation can play in improving social welfare. Indeed, taxes can be efficiently levied when rents are at stake: in the case here investigated this solution is aimed at limiting the appropriability of rents granted by IPRs. Governments precisely tax profits deriving from IPRs, and patentsbut also trademarks and copyrights - are becoming a more and more fundamental component of assets of corporations. Taxation in this field, however, is often avoided by multinational corporations through the resort to various techniques. The registration of patents can, e.g., be made by a subsidiary operating in a country applying low tax rates. This affiliate then collects profits from royalties, often paid by other firms belonging to the same corporation which apply inflated transfer prices in order to shift profits to the low tax country (Karkinsky and Riedel [2012]). Governments can resort to taxation schemes that close such loopholes, through stricter rules about the imputation of profits at the corporation's residence country. ${ }^{16}$ The recent public economics literature depicts taxation of capital income (capital in this framework, unlike in our model, includes IPRs) in a more favorable way than in the past (see, e.g., Diamond and Saez [2011], or, for a dynamic setting, Aghion et al. [2013]), as part of a strategy for fighting also other forms of tax avoidance and for improving equity and efficiency.

that of sustained growth when $\tau=0$ (equal to 3.276180871 ) by an order of $10^{-9}$.

${ }^{16}$ The CFC (Controlled Foreign Company) rule makes patent income earned by subsidiaries in low tax countries taxable at the parent location. It aims at preventing erosion of the home country tax base, by denying the deferral of profit taxation until repatriation. The conditions for rendering the income immediately taxable for the parent company vary from country to country, and usually refer to the characteristics of the country in which the controlled company is located (which can, e.g., be included in a black or gray list of likely tax heavens) or to the type of income. Royalties from patents are often considered taxable as long as they represent passive income, i.e., they just generate a rent and are not directly used for productive purposes by the controlled firm. The OECD is intervening in the filed of IPR income taxation through the Base Erosion and Profit Shifting (BEPS) program (OECD [2013]). 
Among the features that allow the simplified patent system sketched out in this paper to ensure in the long run a convergence to the same growth pattern as that characterizing a fully efficient system, there is the fact that the R\&D outputs bought by the government are immediately made available both to the production sector and to the other researchers, so that the process of knowledge recombination can go on smoothly. The real world differs from the model in the length of patent protection as it implies in many jurisdictions a delay up to 20 years, which thus more severely affects the recombinant process than in our stylized framework. Like in the model, instead, governments are boosting to some extent the demand for R\&D through various forms of financial support. Nonetheless, and somewhat surprisingly, they have by and large embraced an approach that allows researchers in public universities and laboratories to patent their results, thus contradicting the quest for a quick and easy dissemination of new knowledge.

Since the early studies on economic growth, none doubts on the crucial role of technological progress and knowledge accumulation in determining the long-run economic performance. Debated and delicate is instead how knowledge creation should be fostered and what are the consequences of different policies aiming at supporting innovation. In this paper we introduce a market mechanism as part of the recombinant knowledge creation, originally proposed by Weitzman (1998). The government plays a crucial role in our model: it collects taxes from the final producing sector to finance R\&D activities, and relies on a tax-subsidy scheme in order to correct for the inefficiencies generated by the private appropriation process. We show that in the long run, the economy converges towards an (asymptotic) balanced growth path, along which the economic growth rate is independent of the policy parameter, while the IPRs system and the appropriability policy adopted jointly affect the transitional dynamics and therefore social welfare. Because transitional dynamics in recombinant growth models are tough objects, we rely on quantitative methods to perform comparative dynamic analysis. We show that welfare increases with the tax parameter, that is, in the bound on the appropriability via IPRs: stricter tax (weaker appropriability) regimes yield higher social welfare.

\section{Appendix}

\section{A General results}

Proof of Proposition 1. i) Using (14) and (4), condition (15) is equivalent to

$$
\left(\eta^{-1}\right)^{\prime}\left[1 / C_{m}^{\prime}(A)\right]>C_{m}^{\prime}(A) \eta^{-1}\left[1 / C_{m}^{\prime}(A)\right]
$$

Let

$$
x=1 / C_{m}^{\prime}(A) ;
$$

as $A<\infty$ implies $C_{m}^{\prime}(A)<\infty, x>0$ holds true. Substituting (47) into (46) yields

$$
\left(\eta^{-1}\right)^{\prime}(x)>\left[\eta^{-1}(x)\right] / x
$$

which holds true for all $x>0$ under Assumption A.1.

ii) Condition (16) follows immediately by noting that

$$
\lim _{A \rightarrow \infty} \psi(A)=\lim _{A \rightarrow \infty}\left(\eta^{-1}\right)^{\prime}\left[1 / C_{m}^{\prime}(A)\right]=\left(\eta^{-1}\right)^{\prime}(0)=1 / \eta^{\prime}(0)=\lim _{A \rightarrow \infty} \varphi(A),
$$

where the last equality is (5) in Tsur and Zemel (2007). 
Proof of Proposition 2. By Assumption A.1 $\psi(A)=\left(\eta^{-1}\right)^{\prime}\left[1 / C_{m}^{\prime}(A)\right]$ is decreasing in $A$, so that, by (49), $\psi(A)>\lim _{A \rightarrow \infty} \psi(A)=1 / \eta^{\prime}(0)$ for all $A<\infty$; hence, (7) holds. Plugging the optimal effort $J^{*}$ as in (9) into (17) yields:

$$
\begin{aligned}
\psi(A) \dot{A}-J^{*} & =\psi(A) \dot{A}-H\left(\eta^{\prime}\right)^{-1}[1 / \psi(A)] \\
& =\psi(A) \dot{A}-C_{m}^{\prime}(A) \dot{A}\left(\eta^{\prime}\right)^{-1}[1 / \psi(A)] \\
& =\psi(A) \dot{A}-C_{m}^{\prime}(A) \dot{A}\left(\eta^{\prime}\right)^{-1}\left\{\eta^{\prime}\left[\eta^{-1}\left(1 / C_{m}^{\prime}(A)\right)\right]\right\} \\
& =\psi(A) \dot{A}-C_{m}^{\prime}(A) \dot{A}\left\{\eta^{-1}\left[1 / C_{m}^{\prime}(A)\right]\right\} \\
& =[\psi(A)-\varphi(A)] \dot{A},
\end{aligned}
$$

where in the third equality we used (2), in the fourth we used the first equality in (14), and in the sixth we used (4). By Proposition 1 (i) $[\psi(A)-\varphi(A)] \dot{A}>0$ for all $A<\infty$ when there is knowledge growth (i.e., a positive amount of new knowledge, $\dot{A}$, is produced and sold to the regulator).

To prove Proposition 3 we first need the following lemma.

Lemma 1 Under Assumption A.1 $\varphi(A)$ defined in (4) satisfies:

$$
\lim _{A \rightarrow \infty} \varphi^{\prime}(A) A=0 .
$$

Proof. To establish (50) we show that $\varphi^{\prime}(A)$ is asymptotically equivalent to $k / A^{2}, \varphi^{\prime}(A) \sim$ $k / A^{2}$, for $A \rightarrow \infty$, where $k$ is some constant, which implies that $\varphi^{\prime}(A)=o(A)$ for $A \rightarrow \infty$. Differentiating the RHS in (4) with respect to $A$ we easily get

$$
\varphi^{\prime}(A)=C_{m}^{\prime \prime}(A)\left\{\eta^{-1}\left[\frac{1}{C_{m}^{\prime}(A)}\right]-\frac{1}{C_{m}^{\prime}(A)}\left(\eta^{-1}\right)^{\prime}\left[\frac{1}{C_{m}^{\prime}(A)}\right]\right\} .
$$

From the definition $C_{m}(A)=A ! /[m !(A-m) !]$ it is straightforward to check that $C_{m}(A) \sim$ $A^{m}, C_{m}^{\prime}(A) \sim A^{m-1}, C_{m}^{\prime \prime}(A) \sim A^{m-2}$, and so on for $A \rightarrow \infty$. Hence, setting $x=1 / C_{m}^{\prime}(A)$ we have that $x \rightarrow 0^{+}$as $A \rightarrow \infty$ and we can use the second and first order MacLaurin expansions of $\eta^{-1}(x)$ and $\left(\eta^{-1}\right)^{\prime}(x)$ around $0^{+}$respectively:

$$
\begin{aligned}
\eta^{-1}(x) & =\eta^{-1}(0)+\left(\eta^{-1}\right)^{\prime}(0) x+\frac{1}{2}\left(\eta^{-1}\right)^{\prime \prime}(0) x^{2}+o\left(x^{2}\right) \text { for } x \rightarrow 0^{+} \\
\left(\eta^{-1}\right)^{\prime}(x) & =\left(\eta^{-1}\right)^{\prime}(0)+\left(\eta^{-1}\right)^{\prime \prime}(0) x+o(x) \text { for } x \rightarrow 0^{+} .
\end{aligned}
$$

Noting that, by Assumption A.1, $\eta^{-1}(0)=0$ and both $\left(\eta^{-1}\right)^{\prime}(0)$ and $\left(\eta^{-1}\right)^{\prime \prime}(0)$ have finite values we rewrite (51) accordingly:

$$
\begin{aligned}
\varphi^{\prime}(A)= & C_{m}^{\prime \prime}(A)\left\{\frac{\left(\eta^{-1}\right)^{\prime}(0)}{C_{m}^{\prime}(A)}+\frac{1}{2} \frac{\left(\eta^{-1}\right)^{\prime \prime}(0)}{C_{m}^{\prime}(A)^{2}}+o\left[\frac{1}{C_{m}^{\prime}(A)^{2}}\right]\right. \\
& \left.-\frac{\left(\eta^{-1}\right)^{\prime}(0)}{C_{m}^{\prime}(A)}-\frac{\left(\eta^{-1}\right)^{\prime \prime}(0)}{C_{m}^{\prime}(A)^{2}}-\frac{1}{C_{m}^{\prime}(A)} o\left[\frac{1}{C_{m}^{\prime}(A)}\right]\right\} \text { for } A \rightarrow \infty \\
= & C_{m}^{\prime \prime}(A)\left\{-\frac{1}{2} \frac{\left(\eta^{-1}\right)^{\prime \prime}(0)}{C_{m}^{\prime}(A)^{2}}+o\left[\frac{1}{C_{m}^{\prime}(A)^{2}}\right]\right\} \text { for } A \rightarrow \infty,
\end{aligned}
$$


where in the second equality we simplified common terms and used the properties $f(x) o[g(x)]=$ $o[f(x) g(x)]$ and $o[g(x)] \pm o[g(x)]=o[g(x)]$. Using the asymptotic equivalences $C_{m}^{\prime}(A) \sim$ $A^{m-1}$ and $C_{m}^{\prime \prime}(A) \sim A^{m-2}$ for $A \rightarrow \infty$ we can write

$$
\varphi^{\prime}(A) \sim A^{m-2}\left[-\frac{1}{2} \frac{\left(\eta^{-1}\right)^{\prime \prime}(0)}{A^{2(m-2)}}+o\left(\frac{1}{A^{2(m-2)}}\right)\right] \text { for } A \rightarrow \infty,
$$

which is equivalent to

$$
\varphi^{\prime}(A) \sim \frac{A^{m-2}}{A^{2(m-2)}}\left[-\frac{1}{2}\left(\eta^{-1}\right)^{\prime \prime}(0)+o(1)\right]=\frac{k}{A^{2}} \text { for } A \rightarrow \infty
$$

where in the last inequality we used the fact that, under Assumption A.1, $k=-(1 / 2)\left(\eta^{-1}\right)^{\prime \prime}(0)$ is a finite number.

Proof of Proposition 3. i) Assume that $A_{0} \geq A_{\tau}^{s k}$. From Proposition 1(ii) and (20) it is immediately seen that $\lim _{t \rightarrow+\infty} \phi_{\tau}[A(t)]=\lim _{A \rightarrow \infty} \varphi(A)=1 / \eta^{\prime}(0)$ for every $0 \leq \tau \leq 1$. Hence, under sustained growth of knowledge-i.e., when $\lim _{t \rightarrow \infty} A(t)=\infty$-taking the limit as $t \rightarrow \infty$ on the RHS of (30) yields the RHS of (32). The second inequality in (31) implies that, according to $(32), \gamma^{*}=\dot{c} / c>0$. Differentiating with respect to time $\ln (Y)$, where, according to (5), $Y=A f(X / A)$, and recalling that from (22) it follows that $X / A \equiv\left(f^{\prime}\right)^{-1}(1)$ is constant, it is immediately seen that $\dot{Y} / Y=\dot{A} / A$. Dividing the dynamic constraint in (24) by $A$ one gets

$$
\frac{\dot{A}}{A}=\frac{1}{\phi_{\tau}(A)}\left(\delta-\frac{c}{A}\right) \rightarrow \eta^{\prime}(0)\left(\delta-\frac{c}{A}\right) \quad \text { as } A \rightarrow \infty,
$$

which implies that $\dot{A} / A$ can be constant along the BGP only if the ratio $c / A$ on the RHS is constant as well, which is possible if and only if $\dot{A} / A=\dot{c} / c=\gamma^{*}$.

In order to satisfy the transversality condition (28), $\dot{\lambda} / \lambda+\dot{A} / A<\rho$ must hold asymptotically. From (29) we have:

$$
\frac{\dot{\lambda}}{\lambda}+\frac{\dot{A}}{A}=\frac{\phi_{\tau}^{\prime}(A) \dot{A}}{\phi_{\tau}(A)}-\varepsilon_{u}(c) \frac{\dot{c}}{c}+\frac{\dot{c}}{c} .
$$

Condition (16) and the definition of $\phi_{\tau}(A)$ in (20) imply that $\phi_{\tau}(A) \sim \varphi(A)$ for large $A$ values; similarly, $\phi_{\tau}^{\prime}(A) \sim \varphi^{\prime}(A)$ for $A \rightarrow \infty$, or, equivalently, $\phi_{\tau}^{\prime}[A(t)] \sim \varphi^{\prime}[A(t)]$ for $t \rightarrow \infty$. Moreover, $\dot{A}(t) \sim \gamma^{*} A(t)$ for $t \rightarrow \infty$, so that the first term on the RHS in (53) turns out to be asymptotically equivalent to $\varphi^{\prime}[A(t)] \gamma^{*} A(t) / \varphi[A(t)]$ for $t \rightarrow \infty$. We can thus apply Lemma 1 to obtain

$$
\lim _{t \rightarrow \infty} \frac{\phi_{\tau}^{\prime}[A(t)] \dot{A}}{\phi_{\tau}[A(t)]}=\lim _{t \rightarrow \infty} \frac{\varphi^{\prime}[A(t)] \gamma^{*} A(t)}{\varphi[A(t)]}=0,
$$

which, together with the assumption $\lim _{t \rightarrow+\infty} \varepsilon_{u}(c)=\sigma$, implies that

$$
\lim _{t \rightarrow \infty}\left[\frac{\dot{\lambda}(t)}{\lambda(t)}+\frac{\dot{A}(t)}{A(t)}\right]=(1-\sigma) \gamma^{*}=\frac{1-\sigma}{\sigma}\left[\delta \eta^{\prime}(0)-\rho\right] .
$$

Hence, the transversality condition (28) is satisfied whenever

$$
\frac{1-\sigma}{\sigma}\left[\delta \eta^{\prime}(0)-\rho\right]<\rho \quad \Longrightarrow \quad(1-\sigma) \delta \eta^{\prime}(0)<\rho
$$

which is the first inequality in (31). 
ii) The property that the ABGP equilibrium characterized by (32) is Pareto optimal is again a consequence of Proposition 1(ii) and the definition of $\phi_{\tau}(A)$ in (20), which imply that asymptotically new knowledge is being produced at its (constant) cost $\lim _{t \rightarrow+\infty} \phi_{\tau}[A(t)]=$ $\lim _{A \rightarrow \infty} \varphi(A)=1 / \eta^{\prime}(0)$.

iii) Condition (52) implies that, asymptotically,

$$
\frac{c}{A}=\delta-\frac{\gamma^{*}}{\eta^{\prime}(0)}=\delta-\frac{\delta \eta^{\prime}(0)-\rho}{\sigma \eta^{\prime}(0)}
$$

which, after, some algebra, leads to (33).

\section{B Numerical Methods and Social Welfare Calculation}

To numerically estimate the optimal transition trajectories eventually leading to an ABGP defined by the system of ODEs (42) and thoroughly described in Section 5 we follow closely the method pursued in Mulligan and Sala-i-Martin (1991). We first consider the policy function $\tilde{c}_{\tau}(A)$ as the solution of the unique ODE given by the ratio between the two equations in (42):

$$
\begin{aligned}
c^{\prime}(A) & =\frac{\left[\delta-\rho \phi_{\tau}(A)\right]}{\sigma[\delta A-c(A)]} c(A) \\
& =\frac{\left[(1-\alpha) \theta^{1 /(1-\alpha)} \alpha^{\alpha /(1-\alpha)} \beta(2 A-3)^{2}-\rho(2 A-1)(2 A-2 \tau-1)\right]}{\sigma\left[(1-\alpha) \theta^{1 /(1-\alpha)} \alpha^{\alpha /(1-\alpha)} A-c(A)\right] \beta(2 A-3)^{2}} c(A) .
\end{aligned}
$$

To estimate the solution of (54) we apply the standard Fehlberg fourth-fifth order Runge-Kutta method with degree four interpolant available in Maple 2015, using as boundary condition the consumption value given by the linear asymptotic policy function defined in (33). ${ }^{17}$ Specifically, we set a knowledge stock amount deemed sufficiently large to assume that the economy is already on the ABGP, $A_{T}=100000$, so that the optimal policy is given by (33) and the value $c^{*}\left(A_{T}\right)=(1 / \sigma)[(\sigma-1) \delta+\rho / \beta] A_{T}=29404.1649$ can be safely used as boundary (terminal) condition. We then set such value as boundary condition in the Runge-Kutta method and run it backward until a lower bound is found below which the algorithm stops functioning: $A_{0}=4.0107>3 / 2$ turns out to be such lower bound common to all values $\tau=0,0.1, \ldots, 1$, i.e., we assume that a stock of slightly more than 4 successful ideas is available at the beginning of our economy's history. For the parameters' values in (43) this bound is reached for the smallest $\tau$-value, $\tau=0$, corresponding to the maximum rent appropriability by $R \& D$-firms. Hence, all our optimal policy estimations hold on the range $\left[A_{0}, A_{T}\right]=[4.0107,100000]$.

Next, the time-path trajectories for the stock of knowledge $A(t)$ are estimated by replacing the approximated optimal values $\tilde{c}_{\tau}(A)$ just obtained into the first ODE in (42) so to obtain ODEs in the only variable $A(t)$ which again can be solved through the Runge-Kutta algorithm with respect to time $t$. As initial condition we use $A_{0}=4.0107$ and perform the algorithm up to a terminal time $T$ after which the procedure stops because it reaches the maximum knowledge stock considered, $A_{T}=100000$. Such upper bound turns out to be reached soonest under the first-best IPRs policy, $\tau=1$, for which a time range $T=573.7$ is necessary. Time-path trajectories for total output are computed, according to (39), as $y(t)=\theta\left[(\theta \alpha)^{1 /(1-\alpha)}\right]^{\alpha} A(t)=$

\footnotetext{
${ }^{17} \mathrm{~A}$ thorough discussion on the Runge-Kutta method to approximate the solution of an ODE can be found in Chapter 10 of Judd (1998). Note, however, that rather than using the asymptotic steady state (or the ABGP) as boundary condition, Judd adopts a different procedure called "shooting", which consists in guessing an initial optimal value $\tilde{c}(0)$ and checking whether the trajectory thus generated converges toward the asymptotic target. See, e.g., Algorithm 10.3 on p. 357 for the classical Ramsey optimal growth model.
} 
$0.1911 A(t)$, where $(\theta \alpha)^{\alpha /(1-\alpha)} \equiv X(t) / A(t)$ is the (constant) ratio between net output, $X(t)$, and the stock of knowledge, $A(t)$. To generate the optimal consumption time-path trajectories associated to the $A(t)$ dynamics we just evaluate each optimal policy policy $\tilde{c}_{\tau}(A)$ at $A(t)$, i.e., we set $\tilde{c}_{\tau}(t)=\tilde{c}_{\tau}[A(t)]$ for each instant $t \in[0,573.7]$.

Finally, following Privileggi (2015), social welfare is directly estimated through the HJB equation for the value function $V_{\tau}\left(A_{0}\right)$, considered as a function of the initial stock of knowledge $A_{0}$. Note that, to this purpose, only the estimated value of the optimal policy $\tilde{c}_{\tau}(A)$ solving (54) at the initial value $A_{0}, \tilde{c}_{\tau}\left(A_{0}\right)$, is required; that is, neither further numeric approximations of time-path trajectories nor quadrature methods to estimate the integral in (23) on such trajectories are necessary: the only numerical step involved in the whole analysis is the solution estimation of (54). To pursue this approach we need to (slightly) further restrict the assumption on the initial value $A_{0}$, according to the following proposition.

Proposition 4 Under Assumptions A.2-4 and provided that condition (31) of Proposition 3 holds, namely, $(1-\sigma) \beta(1-\alpha) \theta^{1 /(1-\alpha)} \alpha^{\alpha /(1-\alpha)}<\rho<\beta(1-\alpha) \theta^{1 /(1-\alpha)} \alpha^{\alpha /(1-\alpha)}$, if, in addition, $A_{0} \geq 1 / \delta$, then the value function of problem (23) subject to (24) as a function of any (initial) stock of knowledge $A_{0} \geq 1 / \delta$ is given by

$$
\begin{aligned}
V_{\tau}\left(A_{0}\right) & =\frac{1}{\rho}\left[\frac{\tilde{c}_{\tau}\left(A_{0}\right)^{1-\sigma}-1}{1-\sigma}+\frac{\delta A_{0}-\tilde{c}_{\tau}\left(A_{0}\right)}{\tilde{c}_{\tau}\left(A_{0}\right)^{\sigma}}\right] \\
& =\frac{1}{\rho}\left[\frac{\tilde{c}_{\tau}\left(A_{0}\right)^{1-\sigma}-1}{1-\sigma}+\frac{(1-\alpha) \theta^{1 /(1-\alpha)} \alpha^{\alpha /(1-\alpha)} A_{0}-\tilde{c}_{\tau}\left(A_{0}\right)}{\tilde{c}_{\tau}\left(A_{0}\right)^{\sigma}}\right],
\end{aligned}
$$

where $\tilde{c}_{\tau}\left(A_{0}\right)$ is the solution of (54) evaluated at $A=A_{0}$.

To prove Proposition 4 we first need an ad-hoc verification principle to show that the RHS in (55) actually is the value function of (23), which is the purpose of the following Lemma 2. Given a technology set $T \subseteq \mathbb{R}^{2 n}$, consider the standard continuous-time problem

$$
V\left(x_{0}\right)=\sup \int_{0}^{\infty} e^{-\rho t} U[x(t), \dot{x}(t)] \mathrm{dt}
$$

subject to $[x(t), \dot{x}(t)] \in T$ for all $t \geq 0$ and $x(0)=x_{0}$,

where $\rho>0$ is the discount rate and $U(\cdot, \cdot)$ is the instantaneous felicity. We shall denote by $x\left(t ; x_{0}\right)$ any feasible path starting from $x_{0}$, namely, satisfying $\left[x\left(t ; x_{0}\right), \dot{x}\left(t ; x_{0}\right)\right] \in T$ for all $t \geq 0$. We denote by $T(x)$ the $x$-section of the set $T$, i.e., $T(x)=\left\{(x, \dot{x}) \in \mathbb{R}^{2 n}:(x, \dot{x}) \in T\right\}$.

Lemma 2 (A verification principle) Assume that a function $w: \mathbb{R}^{n} \rightarrow \mathbb{R}$ exists such that:

(i) $w(x) \geq 0$ for all feasible $x$;

(ii) $w(x)$ is of class $C^{1}$ and satisfies the Hamilton-Jacobi-Bellman equation, i.e.,

$$
\rho w(x)=\max _{\dot{x} \in T(x)}[U(x, \dot{x})+\nabla w(x) \cdot \dot{x}]
$$

(iii) for every initial condition $x_{0}$ there is a feasible path $\left[x^{*}\left(t ; x_{0}\right), \dot{x}^{*}\left(t ; x_{0}\right)\right]$ such that the max is attained in (57), i.e.,

$$
\rho w\left[x^{*}\left(t ; x_{0}\right)\right]=U\left[x^{*}\left(t ; x_{0}\right), \dot{x}^{*}\left(t ; x_{0}\right)\right]+\nabla w\left[x^{*}\left(t_{0} ; x_{0}\right)\right] \cdot \dot{x}^{*}\left(t ; x_{0}\right)
$$

for all $t \geq 0$, a.e.; 
(iv) $\lim _{t \rightarrow \infty} e^{-\rho t} w\left[x^{*}\left(t ; x_{0}\right)\right]=0$ for each solution $\left[x^{*}\left(t ; x_{0}\right), \dot{x}^{*}\left(t ; x_{0}\right)\right]$ of $(58)$.

Then $w(x)$ is the value function of (56), i.e., $V(x)=w(x)$, and $\left[x^{*}\left(t ; x_{0}\right), \dot{x}^{*}\left(t ; x_{0}\right)\right]$ is an optimal path, i.e., $V\left(x_{0}\right)=\int_{0}^{\infty} e^{-\rho t} U\left[x^{*}\left(t ; x_{0}\right), \dot{x}^{*}\left(t ; x_{0}\right)\right] \mathrm{dt}$.

Proof. Fix $x_{0}$ and let $[x(t), \dot{x}(t)] \equiv\left[x\left(t ; x_{0}\right), \dot{x}\left(t ; x_{0}\right)\right]$ be any feasible path. In view of (57), at any instant $t$,

$$
\begin{gathered}
\rho w[x(t)] \geq U[x(t), \dot{x}(t)]+\nabla w[x(t)] \cdot \dot{x}(t) \\
\Longleftrightarrow \rho w[x(t)]-\nabla w[x(t)] \cdot \dot{x}(t) \geq U[x(t), \dot{x}(t)] \\
\Longleftrightarrow e^{-\rho t} \rho w[x(t)]-e^{-\rho t} \nabla w[x(t)] \cdot \dot{x}(t) \geq e^{-\rho t} U[x(t), \dot{x}(t)] \\
\Longleftrightarrow-\frac{\partial}{\partial t}\left\{e^{-\rho t} w[x(t)]\right\} \geq e^{-\rho t} U[x(t), \dot{x}(t)],
\end{gathered}
$$

which, integrating both sides, yields

$$
\begin{aligned}
& -\int_{0}^{T} \frac{\partial}{\partial t}\left\{e^{-\rho t} w[x(t)]\right\} \mathrm{dt} \geq \int_{0}^{T} e^{-\rho t} U[x(t), \dot{x}(t)] \mathrm{dt} \\
& \Longleftrightarrow w[x(0)]-e^{-\rho T} w[x(T)] \geq \int_{0}^{T} e^{-\rho t} U[x(t), \dot{x}(t)] \mathrm{dt} \\
& \Longleftrightarrow w\left(x_{0}\right) \geq \int_{0}^{T} e^{-\rho t} U[x(t), \dot{x}(t)] \mathrm{dt}+e^{-\rho T} w[x(T)] .
\end{aligned}
$$

Taking the limit as $t \rightarrow \infty$ in both sides and using assumption (i) we get

$$
w\left(x_{0}\right) \geq \int_{0}^{\infty} e^{-\rho t} U[x(t), \dot{x}(t)] \mathrm{d} t
$$

which, as $[x(t), \dot{x}(t)]$ is arbitrary, implies that $w\left(x_{0}\right) \geq V\left(x_{0}\right)$.

Repeating all steps above with a feasible path $\left[x^{*}(t), \dot{x}^{*}(t)\right] \equiv\left[x^{*}\left(t ; x_{0}\right), \dot{x}^{*}\left(t ; x_{0}\right)\right]$ satisfying (58) one easily gets to

$$
w\left(x_{0}\right)=\int_{0}^{T} e^{-\rho t} U\left[x^{*}(t), \dot{x}^{*}(t)\right] \mathrm{dt}+e^{-\rho T} w\left[x^{*}(T)\right],
$$

which, taking the limit as $t \rightarrow \infty$ in both sides and using assumption (iv), leads to

$$
w\left(x_{0}\right)=\int_{0}^{\infty} e^{-\rho t} U\left[x^{*}(t), \dot{x}^{*}(t)\right] \mathrm{dt} \leq V\left(x_{0}\right),
$$

where the last inequality holds by definition of value function. Hence, $w\left(x_{0}\right)=V\left(x_{0}\right)$ and, clearly, $\left[x^{*}(t), \dot{x}^{*}(t)\right]$ is an optimal path.

Proof of Proposition 4. We must show that assumptions (i)-(iv) of Lemma 2 hold for the function defined in (55), namely,

$$
w(A)=\frac{1}{\rho}\left[\frac{\tilde{c}_{\tau}(A)^{1-\sigma}-1}{1-\sigma}+\frac{\delta A-\tilde{c}_{\tau}(A)}{\tilde{c}_{\tau}(A)^{\sigma}}\right],
$$

where $\delta=\beta(1-\alpha) \theta^{1 /(1-\alpha)} \alpha^{\alpha /(1-\alpha)}$. 
To check (i) it is convenient to rewrite (55) as

$$
w(A)=\frac{1}{\rho}\left(\frac{\sigma c^{1-\sigma}-1}{1-\sigma}+\frac{\delta A}{c^{\sigma}}\right) ;
$$

taking the derivative with respect to $c$, yields

$$
\frac{\partial}{\partial c} w(A)=\frac{\sigma}{\rho c^{\sigma}}\left(1-\frac{\delta A}{c}\right)
$$

which, as $c \leq \delta A$ must hold (consumption cannot exceed net output), is clearly non positive, namely, $w(A)$ is decreasing in consumption. Hence, to show that $w(A) \geq 0$ it is sufficient to check that it is positive for the largest admissible consumption, namely, $c=\delta A$ (corresponding to no growth, as no investment in R\&D is performed); substituting it into (59) we get

$$
w(A)=\frac{(\delta A)^{1-\sigma}-1}{\rho(1-\sigma)},
$$

which is clearly non negative whenever $\delta A \geq 1$, that is, $A \geq 1 / \delta$.

Using the resource constraint (21) and (19), the social planner optimal consumption can be written as $\tilde{c}_{\tau}(A)=\delta A-G=\delta A-\phi_{\tau}(A) \dot{A}$, so that the reduced-form utility as in (56) is:

$$
U(A, \dot{A})=\frac{\left[\delta A-\phi_{\tau}(A) \dot{A}\right]^{1-\sigma}-1}{1-\sigma} .
$$

Hence, the RHS in (57) turns out to be

$$
\frac{\left[\delta A-\phi_{\tau}(A) \dot{A}\right]^{1-\sigma}-1}{1-\sigma}+w^{\prime}(A) \dot{A},
$$

which is clearly strictly concave in $\dot{A}$; hence, there is a unique value $\dot{A}^{*}$ such that

$$
-\left[\delta A-\phi_{\tau}(A) \dot{A}^{*}\right]^{-\sigma} \phi_{\tau}(A)+w^{\prime}(A)=0
$$

satisfies the FOC and thus attains the maximum in the RHS of (57). If such value $\dot{A}^{*}$ exists for each $A(t)$, then both (ii) and (iii) are satisfied, i.e., there is a feasible path $\left[A^{*}\left(t ; A_{0}\right), \dot{A}^{*}\left(t ; A_{0}\right)\right]$ such that (58) holds:

$$
\rho w\left[A^{*}\left(t ; A_{0}\right)\right]=U\left[A^{*}\left(t ; A_{0}\right), \dot{A}^{*}\left(t ; A_{0}\right)\right]+w^{\prime}\left[A^{*}\left(t ; A_{0}\right)\right] \cdot \dot{A}^{*}\left(t ; A_{0}\right) \quad \text { for all } t \geq 0 .
$$

To this purpose we now show that the path $\left[A^{*}\left(t ; A_{0}\right), \dot{A}^{*}\left(t ; A_{0}\right)\right]$ is the trajectory generated by the optimal policy $\tilde{c}_{\tau}(A)$, that is, according to the dynamic constraint in $(24)$,

$$
\dot{A}^{*}\left(t ; A_{0}\right)=\frac{\delta A^{*}\left(t ; A_{0}\right)-\tilde{c}_{\tau}\left[A^{*}\left(t ; A_{0}\right)\right]}{\phi_{\tau}\left[A^{*}\left(t ; A_{0}\right)\right]} \quad \text { for all } t \geq 0 .
$$

Dropping time dependency in (61), we use it to rewrite (60) as

$$
w^{\prime}(A)=\frac{\phi_{\tau}(A)}{\tilde{c}_{\tau}(A)^{\sigma}}
$$


and show that the derivative with respect to $A$ of the RHS in (55) coincides with (62). Specifically:

$$
\frac{\partial}{\partial A} w(A)=\frac{1}{\rho}\left\{\frac{\tilde{c}_{\tau}^{\prime}(A)}{\tilde{c}_{\tau}(A)^{\sigma}}+\frac{\left[\delta-\tilde{c}_{\tau}^{\prime}(A)\right] \tilde{c}_{\tau}(A)^{\sigma}-\sigma\left[\delta A-\tilde{c}_{\tau}(A)\right] \tilde{c}_{\tau}(A)^{\sigma-1} \tilde{c}_{\tau}^{\prime}(A)}{\tilde{c}_{\tau}(A)^{2 \sigma}}\right\},
$$

which, after some algebra, boils down to

$$
\frac{\partial}{\partial A} w(A)=\frac{1}{\rho \tilde{c}_{\tau}(A)^{\sigma}}\left[\delta-\sigma \frac{\delta A-\tilde{c}_{\tau}(A)}{\tilde{c}_{\tau}(A)} \tilde{c}_{\tau}^{\prime}(A)\right] .
$$

Using the definition of the optimal policy $\tilde{c}_{\tau}(A)$ as the solution of the ODE (54) and replacing $\tilde{c}_{\tau}^{\prime}(A)$ in the above equation accordingly, $(62)$ is immediately obtained and thus $w(A)$ in $(55)$ fully satisfies (ii) and (iii) of Lemma 2.

Finally, from Proposition 3 we know that asymptotically both the optimal consumption $\tilde{c}_{\tau}$ and knowledge $A$ grow at the common constant rate in (32): $\gamma^{*}=\dot{c} / c=\dot{A} / A=(\delta \beta-\rho) / \sigma$, that is, $c(t)=\tilde{c}_{\tau}(0) e^{\gamma^{*} t}$ and $A(t)=A_{0} e^{\gamma^{*} t}$. Substituting these values in (59) we easily get

$$
w[A(t)] \sim-\frac{1}{\rho(1-\sigma)}+\frac{1}{\rho}\left[\frac{\sigma \tilde{c}_{\tau}(0)^{1-\sigma}}{1-\sigma}+\frac{\delta A_{0}}{\tilde{c}_{\tau}(0)^{\sigma}}\right] e^{(1-\sigma) \gamma^{*} t} \text { for } t \rightarrow \infty .
$$

Therefore,

$$
\lim _{t \rightarrow \infty} e^{-\rho t} w[A(t)]=\lim _{t \rightarrow \infty}\left\{-\frac{e^{-\rho t}}{\rho(1-\sigma)}+\frac{1}{\rho}\left[\frac{\sigma \tilde{c}_{\tau}(0)^{1-\sigma}}{1-\sigma}-\frac{1}{1-\sigma}+\frac{\delta A_{0}}{\tilde{c}_{\tau}(0)^{\sigma}}\right] e^{\left[(1-\sigma) \gamma^{*}-\rho\right] t}\right\}=0
$$

because, by (32), $(1-\sigma) \gamma^{*}-\rho<0$ is equivalent to

$$
(1-\sigma) \frac{\delta \beta-\rho}{\sigma}<\rho \quad \Longleftrightarrow \quad(1-\sigma) \delta \beta<\rho,
$$

which is the left inequality in (31). Thus, (iv) is satisfied as well and the proof is complete.

\section{References}

1. Acemoglu, D. and U. Akcigit, "Intellectual Property Rights Policy, Competition and Innovation", Journal of the European Economic Association, 10 (2012), 1-42.

2. Aghion, P., U. Akcigit, and J. Fernández-Villaverde, "Optimal Capital versus Labor Taxation with Innovation-Led Growth", NBER Working Paper No. 19086, 2013.

3. Arrow, K.J., "Economic Welfare and the Allocation of Resources for Invention", in The Rate and Direction of Inventive Activity: Economic and Social Factors, NBER Special Conference Series Vol. 13 (Princeton, NJ: Princeton University Press, 1962).

4. Bessen, J., and E. Maskin, "Sequential Innovation, Patents and Imitation", Rand Journal of Economics, 40 (2009), 611-635.

5. Ceruzzi, P.E., A History of Modern Computing (Cambridge, MA: The MIT Press, 2003). 
6. Chantrel, É., Grimaud, A., and F. Tournemaine, "Pricing Knowledge and Funding Research of New Technology Sectors in a Growth Model", Journal of Public Economic Theory, 14 (2012), 493-520.

7. Cremers, K., Ernicke, M., Gaessler, F., Harhoff, D., Helmers, C., McDonagh, L., Schliesser, P., van Zeebroeck, N., "Patent Litigation in Europe", European Journal of Law and Economics, forthcoming (2016).

8. Denicolò, V., and L. A. Franzoni. "Patents, Secrets, and the First-Inventor Defense", Journal of Economics and Management Strategy, 13 (2004), 517-538.

9. Diamond, P., and E. Saez, "The Case for a Progressive Tax. From Basic Research to Policy Recommendations", Journal of Economic Perspectives, 25 (2011) 169-190.

10. Eicher, T., and C. García-Peñalosa, "Endogenous Strength of Intellectual Property Rights: Implications for Economic Development and Growth", European Economic Review, 52 (2008), 237-258.

11. Gilbert, R.J., and D. Newbery, "Preemptive Patenting and the Persistence of Monopoly", American Economic Review, 72 (1982), 514-526.

12. Hall, B.H., and Ziedonis R.H., "The patent paradox revisited: an empirical study of patenting in the U.S. semiconductor industry, 1979-1995", Rand Journal of Economics, 32 (2001), 101-128.

13. Heller, M., and R. Eisenberg, "Can Patent Deter Innovation? The Anticommons in Biomedical Research", Science, 280 (1998), 698-701.

14. Judd, K.L. Numerical Methods in Economics (Cambridge, MA: MIT Press, 1998).

15. Karkinsky, T., and N. Riedel, "Corporate Taxation and the Choice of Patent Location Within Multinational Firms", Journal of International Economics, 88 (2012), 176-185.

16. Marchese, C., Privileggi, F. "Endogenous economic growth with disembodied knowledge", Journal of Public Economic Theory, forthcoming, 2017

17. Mulligan, C.B., and X. Sala-i-Martin, "A Note on the Time-Elimination Method for Solving Recursive Dynamic Economic Models", NBER Working Paper No. 116, 1991.

18. Mulligan, C.B., and X. Sala-i-Martin, "Transitional Dynamics in Two-Sector Models of Endogenous Growth", The Quarterly Journal of Economics, 108 (1993), 737-773.

19. Organization of Economic Cooperation and Development, Addressing Base Erosion and Profit Shifting (Paris, FR: OECD, 2013).

20. Organization of Economic Cooperation and Development, Main Science and Technology Indicators, Volume 2016, Issue 1 (Paris, FR: OECD, 2016).

21. Palivos, T., P. Wang, and J. Zhang, "On the existence of balanced growth equilibrium", International Economic Review, 38 (1997), 205-224.

22. Privileggi, F., "On the Transition Dynamics in Endogenous Recombinant Growth Models", in Nonlinear Dynamics in Economics, Finance and Social Sciences-Essays in Honour of John Barkley Rosser Jr, G.I. Bischi, C. Chiarella, and L. Gardini, eds. (Berlin Heidelberg, GE: Springer-Verlag, 2010), pp. 251-278. 
23. Privileggi, F., "Transition Dynamics in Endogenous Recombinant Growth Models by means of Projection Methods", Computational Economics, 38 (2011), 367-387.

24. Privileggi, F., "Takeoff vs. Stagnation in Endogenous Recombinant Growth Models", Mathematics and Computers in Simulation, 108 (2015), 184-214.

25. Privileggi, F. and S. Marsiglio, "Dynamics and Welfare in Recombinant Growth Models with Intellectual Property Rights: a Computational Method", Dip. "Cognetti de Martiis" Working Paper No. 14/2014, 2014 (http://www.unito.it/unitoWAR/ShowBinary/... ...FSRepo/D031/Allegati/WP2014Dip/ WP_14_2014.pdf).

26. Ramello, G.B., "Private Appropriability and Knowledge Sharing: Contradiction or Convergence? The Opposite Tragedy of Creative Commons", in Developments in the Economics of Copyright, L. Takeyama, W. Gordon, and R. Towse, eds. (Cheltenham and Northampton, MA: Edward Elgar, 2005).

27. Ramello, G.B., "Access to vs. exclusion from knowledge: Intellectual property, efficiency and social justice", in Intellectual property and theories of justice, A. Gosserie, A. Marciano, and A. Strowel, eds. (London and Basingstoke, UK: Palgrave, 2008).

28. Ramello, G.B., "Property rights and externalities: the uneasy case of knowledge", European Journal of Law and Economics, 31 (2011), 123-141.

29. Romer, P. M., "Endogenous Technological Change", Journal of Political Economy, 98 (1990), S71-S102.

30. Rosenberg, N., Perspectives on technology (Cambridge, MA: Cambridge University Press, 1976).

31. Scotchmer, S., "Standing on the Shoulders of Giants", Journal of Economic Perspectives, 5 (1991), 29-41.

32. Scotchmer, S., Innovation and Incentives (Cambridge, MA: The MIT Press, 2004).

33. Shavell S., and T. van Ypersele, "Rewards versus Intellectual Property Rights", Journal of Law and Economics, 44 (2001), 525-547.

34. Tsur, Y., and A. Zemel, "Towards Endogenous Recombinant Growth", Journal of Economic Dynamics and Control, 31 (2007), 3459-3477.

35. Weitzman, M.L., "Recombinant Growth", Quarterly Journal of Economics 113 (1998), $331-360$.

36. Ziedonis, R.H., "Don’t Fence Me In: Fragmented Market for Technology and the Patent Acquisition Strategies of the Firms", Management Science, 50 (2004), 804-820. 